\title{
If sterile neutrinos exist, how can one determine the total solar neutrino fluxes?
}

\author{
John N. Bahcall, ${ }^{1, *}$ M. C. Gonzalez-Garcia, ${ }^{2,3,4, \dagger}$ and C. Peña-Garay ${ }^{3, ;}$ \\ ${ }^{1}$ School of Natural Sciences, Institute for Advanced Study, Princeton, New Jersey 08540 \\ ${ }^{2}$ Theory Division, CERN, CH-1211 Geneva 23, Switzerland \\ ${ }^{3}$ Instituto de Física Corpuscular, Universitat de València-CSIC, Edificio Institutos de Paterna, Apt 22085, 46071 València, Spain \\ ${ }^{4}$ C. N. Yang Institute for Theoretical Physics, State University of New York at Stony Brook, Stony Brook, New York $11794-3840$
}

(Received 9 May 2002; published 19 September 2002)

\begin{abstract}
The ${ }^{8} \mathrm{~B}$ solar neutrino flux inferred from a global analysis of solar neutrino experiments is within $11 \%(1 \sigma)$ of the predicted standard solar model value if only active neutrinos exist, but could be as large as 1.7 times the standard prediction if sterile neutrinos exist. We show that the total ${ }^{8} \mathrm{~B}$ neutrino flux (active plus sterile neutrinos) can be determined experimentally to about $10 \%(1 \sigma)$ by combining charged current measurements made with the KamLAND reactor experiment and with the SNO CC solar neutrino experiment, provided the LMA neutrino oscillation solution is correct and the simulated performance of KamLAND is valid. Including also SNO NC data, the sterile component of the ${ }^{8} \mathrm{~B}$ neutrino flux can be measured by this method to an accuracy of about $12 \%(1 \sigma)$ of the standard solar model flux. Combining Super-Kamiokande and KamLAND measurements and assuming the oscillations occur only among active neutrinos, the ${ }^{8} \mathrm{~B}$ neutrino flux can be measured to $6 \%(1 \sigma)$; the total flux can be measured to an accuracy of about $9 \%$. The total ${ }^{7} \mathrm{Be}$ solar neutrino flux can be determined to an accuracy of about $28 \%(1 \sigma)$ by combining measurements made with the KamLAND, SNO, and gallium neutrino experiments. One can determine the total ${ }^{7} \mathrm{Be}$ neutrino flux to a $1 \sigma$ accuracy of about $11 \%$ or better by comparing data from the KamLAND experiment and the BOREXINO solar neutrino experiment provided both detectors work as expected. The $p p$ neutrino flux can be determined to about $15 \%$ using data from the gallium, KamLAND, BOREXINO, and SNO experiments.
\end{abstract}

DOI: 10.1103/PhysRevC.66.035802 PACS number(s): 26.65.+t, 14.60.Pq, 12.15.Ff, 13.15. $+\mathrm{g}$

\section{INTRODUCTION}

We describe in this paper analysis procedures that can answer two of the most important questions of neutrino research. How can one determine the total solar neutrino fluxes $\left({ }^{8} \mathrm{~B},{ }^{7} \mathrm{Be}\right.$, and $\left.p p\right)$ for comparison with solar model predictions? How can one determine the sterile contribution to the total solar neutrino fluxes? Our answers allow for the possibility of an arbitrary mixture in solar neutrino oscillations of active and sterile neutrinos, but require the correctness of the LMA solution of the solar neutrino problems and careful attention to all the sources of error (theoretical as well as experimental). ${ }^{1}$

We focus first on determining total fluxes by comparing charged current $(\mathrm{CC})$ observables measured in different ex-

\footnotetext{
*Electronic address: jnb@ias.edu

${ }^{\dagger}$ Electronic address: concepcion.gonzalez-garcia@cern.ch

*Electronic address: penya@ific.uv.es

${ }^{1}$ This paper was originally written and posted on the electronic archive (hep-ph) before the announcements of the recent SNO results $[1,2]$ and the improved SAGE measurement of the gallium rate [3] and also before our paper was submitted for publication. We have included in the analysis reported in this version of the paper, which we are submitting for publication, the recent SNO and SAGE measurements. The ideas with respect to the ${ }^{7} \mathrm{Be}$ and ${ }^{8} \mathrm{~B}$ neutrinos are unchanged and the numerical results have not been affected significantly, but the present version is more up-to-date with respect to the input data. We have also added, inspired by the SAGE discussion, a detailed analysis of what one can learn about $p p$ neutrinos before there is a dedicated experiment to measure just the $p p$ neutrino flux.
}

periments; this method yields results as independent as possible of uncertainties due to the presence of sterile neutrinos. We then describe how similar techniques can be applied to determine total solar neutrino fluxes using a $\mathrm{CC}$ experiment plus a neutrino-electron scattering experiment [or a neutral current (NC) measurement], which yields results that depend more on the sterile neutrino mix but which can nevertheless be relatively accurate.

The numerical values we estimate for the expected precision with which different quantities can be measured rely upon simulations of the performance of the relevant experiments. Therefore the accuracies that we quote are illustrative; the actual accuracies that are obtainable can only be determined once the experimental uncertainties are known.

\section{A. Flavor changes occur}

Neutrinos change flavors as they travel to the Earth from the center of the Sun. This flavor change was seen directly by the comparison of the Sudbury Neutrino Observatory (SNO) measurement [4] of the charged current reaction for ${ }^{8} \mathrm{~B}$ solar neutrinos with the Super-Kamiokande measurement [5] of the neutrino-electron scattering rate (charged plus neutral current). Even more clearly, flavor change has been demonstrated by comparing the neutral current measurement by SNO with the SNO CC measurement [1]. The conclusion that flavor changes occur among solar neutrinos, if based solely upon the comparison of the SNO and SuperKamiokande event rates, is valid statistically at about the $3.2 \sigma$ confidence level $[4,6-9]$. The neutral current measurement of SNO increases the significance level for flavor changes among solar neutrinos to the $5.3 \sigma$ confidence level. 
The SNO and Super-Kamiokande results demonstrate simply that new physics is required to resolve the longstanding solar neutrino problem [10], i.e., to understand the origin of the discrepancy between the predictions of the standard solar model [11] and the observed solar neutrino event rates $[4,5,12-16]$. If one includes the results of the Chlorine [12], Kamiokande [13], SAGE [14], GALLEX [15], and GNO [16] experiments together with the SNO (CC) and Super-Kamiokande results, then the combined measurements require [9] new physics at $4.0 \sigma$ and, if the relative temperature scaling of the ${ }^{7} \mathrm{Be}$ and ${ }^{8} \mathrm{~B}$ neutrino production reactions is taken into account, at 7.4 $\mathrm{\sigma}$. Helioseismological measurements confirm the predicted sound speeds of the Standard Solar Model to better than $0.1 \%$ and show that stellar physics cannot account for the discrepancies between standard predictions and the observed solar neutrino rates [17].

\section{B. Current knowledge of the ${ }^{8} B$ solar neutrino flux if only active neutrinos exist}

The combination of the charged current (CC) and the charged plus neutral-current measurement with SuperKamiokande has been used by several groups $[4,6-8]$ to determine the flux of active ${ }^{8} \mathrm{~B}$ neutrinos independent of the solar model. These model-independent determinations of the active flux exploit the similarity between the response functions in the SNO and Super-Kamiokande detectors $[4,6,18,19]$. In addition, if one includes all the experimental data in a global oscillation solution in which the ${ }^{8} \mathrm{~B}$ flux is a free parameter, one obtains a similar (but slightly smaller) allowed range for the ${ }^{8} \mathrm{~B}$ neutrino flux [20-22]. The measurement of the $\mathrm{NC}$ rate by SNO [1] provides an independent determination of the active ${ }^{8} \mathrm{~B}$ neutrino flux.

All of the analyses yield the same result: if electron neutrinos oscillate into only active neutrinos, then the total ${ }^{8} \mathrm{~B}$ neutrino flux is in excellent agreement with the flux predicted by the standard solar model.

This close agreement of the active ${ }^{8} \mathrm{~B}$ neutrino flux with the total flux predicted by the standard solar model (SSM) $[11,22]$ is, if the flux of sterile neutrinos is small, an important confirmation of the quantitative theory of stellar evolution. We summarize below the current best-estimates and the associated $1 \sigma$ uncertainties for the active ${ }^{8} \mathrm{~B}$ neutrino flux $\phi_{\text {active }}\left({ }^{8} \mathrm{~B}\right)$.

Standard solar model (BP00) prediction $[11]:^{2}$

$$
\phi_{\text {active }}\left({ }^{8} \mathrm{~B}\right)=5.05 \times 10^{6} \mathrm{~cm}^{2} \mathrm{~s}^{-1}\left(1_{-0.16}^{+0.20}\right) .
$$

Comparison of SNO and Super-Kamiokande event rates (see Ref. [4]):

$$
\phi_{\text {active }}\left({ }^{8} \mathrm{~B}\right)=5.44 \times 10^{6} \mathrm{~cm}^{2} \mathrm{~s}^{-1}(1 \pm 0.18) .
$$

SNO neutral current measurement (assuming undistorted spectrum) [1]:

\footnotetext{
${ }^{2}$ The recently measured low-energy cross section factor reported by Junghans et al. [23] is currently being reinvestigated.
}

TABLE I. The allowed range of the total ${ }^{8} \mathrm{~B}$ neutrino flux. The table presents the allowed range of $f_{B}$ [defined by Eq. (7)] that was found in a global solution of all the currently available solar and reactor neutrino data. The second column gives the allowed range of $f_{B}$ for an arbitrary mixture of active and sterile neutrinos and the third column gives the range for the case where only active neutrinos are considered. The results shown were obtained using Eq. (8).

\begin{tabular}{ccc}
\hline \hline C.L. & $f_{B}($ active + sterile $)$ & $f_{B}$ (active) \\
\hline $1 \sigma$ & $0.99-1.25$ & $0.99-1.15$ \\
$2 \sigma$ & $0.92-1.47$ & $0.92-1.22$ \\
$3 \sigma$ & $0.84-1.67$ & $0.84-1.29$ \\
\hline \hline
\end{tabular}

$$
\phi_{\text {active }}\left({ }^{8} \mathrm{~B}\right)=5.09 \times 10^{6} \mathrm{~cm}^{2} \mathrm{~s}^{-1}(1 \pm 0.12) .
$$

Global neutrino oscillation analysis (see Table I this paper and Ref. [22]):

$$
\phi_{\text {active }}\left({ }^{8} \mathrm{~B}\right)=5.40 \times 10^{6} \mathrm{~cm}^{2} \mathrm{~s}^{-1}(1 \pm 0.075) .
$$

The agreement, summarized in Eqs. (1)-(4), between the SSM calculated flux and the measured active flux suggests that the sterile neutrino contribution to the ${ }^{8} \mathrm{~B}$ neutrino flux may be small. In this paper, we ignore this tempting suggestion and instead concentrate on developing methods to determine experimentally the total ${ }^{8} \mathrm{~B}$ and ${ }^{7} \mathrm{Be}$ neutrino fluxes emitted by the Sun, independent of the active-sterile mixture (for a discussion of earlier investigations of sterile neutrinos see Refs. [24,25]).

If we want to understand the particle physics implications of solar neutrino research, we must determine if sterile neutrinos are present in the solar neutrino flux. Moreover, the original—and still valid — goal of solar neutrino research was [10] to compare solar model predicted and experimentally measured (total) solar neutrino fluxes.

\section{What can one do if sterile neutrinos exist?}

What is the situation if sterile neutrinos exist? The total flux of ${ }^{8} \mathrm{~B}$ neutrinos could in this case be much larger than the standard solar model prediction; a major fraction of the total flux that reaches the Earth could arrive in a form that is not detected in solar neutrino experiments. The existing data

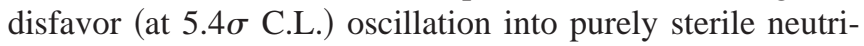
nos. Nevertheless, a large sterile component is allowed $[22,26]$ if oscillations occur into a combination of active and sterile neutrino states (see Refs. [27,28] for a description of the formalism adopted here). The flux of sterile neutrinos could in principle be large enough to destroy the apparently excellent agreement between the flux predicted by the SSM and the true flux of ${ }^{8} \mathrm{~B}$ neutrinos [which is assumed to be pure active neutrinos in the comparison shown above in Eqs. (1)-(4)].

A measurement of the total ${ }^{8} \mathrm{~B}$ solar neutrino flux, including the sterile component (if any), will provide information that is important for astrophysics and for particle physics. The motivation for investigating sterile neutrinos is not dependent upon the LSND [29] results that might suggest the existence of sterile neutrinos. Of course, the LSND results 
are not supported by other experimental results (see Ref. [30]) nor by theoretical predictions that have been precisely confirmed (such as the helioseismological verifications of the standard solar model), as is the case for the inference of flavor change based upon the SNO-Super-Kamiokande comparison.

Fortunately, the KamLAND reactor neutrino experiment [31], when combined with the SNO measurement of the CC flux, is capable of providing a precise determination of the total, i.e., the active plus the sterile, ${ }^{8} \mathrm{~B}$ neutrino flux. We assume throughout this paper the correctness of the currently favored large mixing angle (LMA) solution to the solar neutrino problem. If the LMA solution is not correct, then all global analyses of the available solar and reactor data indicate that either the mass difference $\Delta m^{2}$ or the vacuum mixing angle $\theta$ will be too small to produce a measurable effect in the KamLAND experiment (see, e.g., Refs. $[6-8,20,21,31,32])$. In this case, KamLAND will be unable to provide the information required to determine the total ${ }^{8} \mathrm{~B}$ neutrino flux.

Here is the basic physical idea of the method we propose for measuring the total ${ }^{8} \mathrm{~B}$ solar neutrino flux. For the KamLAND [31] experiment, one will know accurately the flux of antineutrinos from the 17 reactors that contribute significantly to the measured antineutrino events. From measurements of the total event rate and the energy spectrum induced by the surviving $\bar{\nu}_{e}$, the KamLAND experimentalists can determine with precision [31,33-35] the antineutrino propagation parameters $\Delta \bar{m}^{2}$ and $\tan ^{2} \bar{\theta}$. Both the KamLAND measurement and the CC SNO measurements are disappearance experiments for neutrinos (or antineutrinos) of similar energies. For the $\mathrm{CC}$ measurement made with $\mathrm{SNO}$, one does not know the total ${ }^{8} \mathrm{~B}$ neutrino flux created in the Sun. But, assuming conservation of CPT, one can use the propagation parameters $\Delta \bar{m}^{2}$ and $\tan ^{2} \bar{\theta}$ determined by KamLAND and the measured (by SNO) CC rate to solve for the flux that gives the observed result. Summarizing, for the KamLAND experiment one knows the total flux but not the propagation parameters, which are measured. For the SNO CC experiment, one will know (from KamLAND) the propagation parameters and therefore can measure the total flux. ${ }^{3}$

Figure 1 shows the results of a refined global solution for the solar neutrino oscillation parameters that was made (see Sec. II and the Appendix) using all the available solar and reactor data. The figure displays the allowed solar neutrino oscillation contours at $1 \sigma, 2 \sigma$, and $3 \sigma$. The results are obtained by the procedures described most recently in Ref. [22], where we have used in the present paper the analysis strategy (a) (of Ref. [36]) including the 1496 day Super-

\footnotetext{
${ }^{3}$ The method described here is of course more general than the specific application to the KamLAND and SNO experiments. In order to determine the total flux, it is sufficient that one measure a set of observables that do not depend upon the solar neutrino flux (in this paper, the measured quantities in the KamLAND experiment) and a quantity that does depend upon the solar neutrino flux (here, the $\mathrm{CC}$ rate in $\mathrm{SNO}$ ).
}

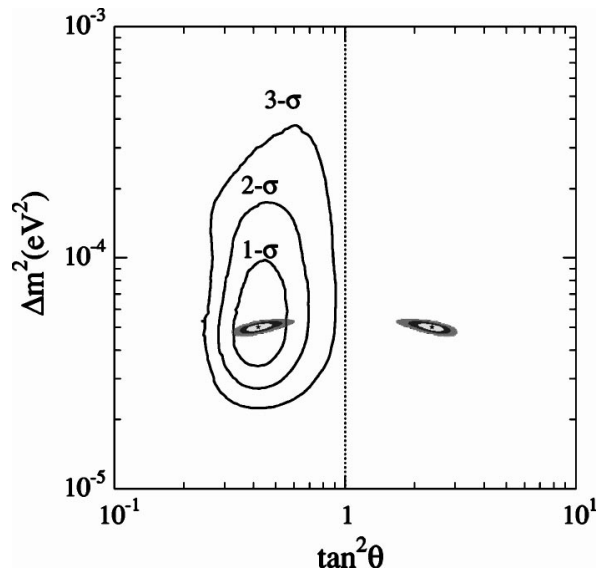

FIG. 1. Solar neutrino allowed region compared with simulated KamLAND allowed region. The figure shows the currently allowed regions of the solar neutrino oscillation parameters; the contours of equal CL are labeled at $1 \sigma, 2 \sigma$, and $3 \sigma$. This global solution was obtained assuming pure active neutrino oscillations and using all the available solar and reactor data. We include the recent SNO results $[1,2]$. The rates from the GALLEX/GNO $[15,16]$ and SAGE $[3,14]$ experiments have been averaged to provide a unique data point (72.4 $\pm 4.7 \mathrm{SNU})$. Some technical improvements regarding neutrino cross sections and correlations of errors were included in the analysis (see the Appendix). The two much smaller allowed regions, placed symmetrically with respect to the line at $\tan ^{2} \theta$ $=1$, represent the allowed regions, at $1 \sigma, 2 \sigma$, and $3 \sigma$, that are obtained from a simulation of what may be achievable with the KamLAND reactor experiment. The best-fit point for the KamLAND simulation is assumed to be the same as the best-fit point for the global solar neutrino oscillation solution, namely, purely active neutrinos with $\Delta m^{2}=5.0 \times 10^{-5} \mathrm{eV}^{2}, \tan ^{2} \theta=0.42$.

Kamiokande data sample [37] as well as the SNO CC, NC, and day-night observations $[1,2]$. We have also made some improvements (see the Appendix) in the treatment of the uncertainties in the neutrino cross sections and in the correlation of errors.

For comparison, we also show in Fig. 1 the small size of the expected allowed region for KamLAND if this reactor antineutrino experiment observes a signal corresponding to the current best-fit point of the solar neutrino analysis. In calculating the KamLAND allowed region, we have made a conservative estimate (see Sec. III C), following the principles discussed in Refs. $[38,39]$. Figure 1 shows clearly that KamLAND has the potential for making a precise measurement of the solar neutrino oscillation parameters, provided that the LMA is the correct oscillation solution.

The only complication involved in determining the total ${ }^{8} \mathrm{~B}$ flux from a comparison of the KamLAND and SNO CC measurements results from the fact that for the favored large mixing angle (LMA) solar neutrino oscillation solution matter effects in the Sun and the Earth can be significant. Matter effects are unimportant for the KamLAND reactor experiment. The role of matter effects in solar neutrino experiments depends somewhat upon the a priori unknown active-sterile mixture, which introduces a calculable uncertainty in the inferred total ${ }^{8} \mathrm{~B}$ neutrino flux. 
In summary, the combination of the SNO CC neutrino measurement and the KamLAND antineutrino measurement will determine the total (active plus sterile) flux $\phi_{\text {total }}\left({ }^{8} \mathrm{~B}\right)$ of ${ }^{8} \mathrm{~B}$ solar neutrinos. By subtracting the previously determined flux (see above), $\phi_{\text {active }}\left({ }^{8} \mathrm{~B}\right)$, from $\phi_{\text {total }}\left({ }^{8} \mathrm{~B}\right)$, one can determine the flux of sterile solar neutrinos.

Using similar reasoning, we shall also show that the combined Super-Kamiokande and KamLAND measurements can be analyzed to yield an accurate value for the total ${ }^{8} \mathrm{~B}$ flux, although in this case the results are somewhat more sensitive to the active-sterile admixture.

\section{D. ${ }^{7}$ Be solar neutrinos}

The flux of ${ }^{7} \mathrm{Be}$ solar neutrinos, which in the SSM is predicted [11] to be $\phi\left({ }^{7} \mathrm{Be}_{\text {active }}\right)=4.77 \times 10^{9} \mathrm{~cm}^{2} \mathrm{~s}^{-1}(1$ \pm 0.10 ), can be determined in a model independent way from a global analysis of the solar neutrino data assuming only active neutrino oscillations. For example, the latest analysis by Garzelli and Giunti [7] yields 0.02 $\leqslant \phi\left({ }^{7} \mathrm{Be}\right) / \phi\left({ }^{7} \mathrm{Be}, \mathrm{SSM}\right) \leqslant 1.15$ at $99 \%$ C.L.

We shall also show in this paper that one can extract the value of the ${ }^{7} \mathrm{Be}$ neutrino flux from measurements of the gallium solar neutrino experiments GALLEX, SAGE, and GNO and the results of the SNO and KamLAND measurements. The value of the ${ }^{7} \mathrm{Be}$ flux that will be derived in this way is relatively insensitive to the assumed neutrino oscillation parameters, although it does depend somewhat on the assumed contributions of the $\mathrm{CNO}$, pep, and $p p$ neutrino fluxes which we adopt from the standard solar model. The constraint provided by the Chlorine experiment is not very useful for determining the total ${ }^{7} \mathrm{Be}$ neutrino flux.

One can obtain an independent measurement of the total ${ }^{7}$ Be solar neutrino flux by comparing data from the KamLAND experiment with data from BOREXINO [40] solar neutrino experiment. If both the KamLAND and the BOREXINO detectors work as expected, then this method will be more accurate than the methods involving the gallium and chlorine radiochemical detectors.

\section{E. Appendix: just for aficionados}

The determination of the total solar neutrino fluxes, and even more so the determination of the sterile components of these neutrino fluxes, requires precision in both the experimental measurements and the theoretical calculations and analyses. We present a refined discussion of the theoretical errors, and their correlations, for the absorption cross for the gallium and Chlorine solar neutrino experiments in the Appendix.

\section{F. Outline and suggested reading strategy}

The outline of this paper is as follows. In Sec. II, we describe the current experimental knowledge of the ${ }^{8} \mathrm{~B}$ solar neutrino flux. Our results are summarized in Table I both for the special case of oscillations between purely active neutrinos and for the general case of oscillations between electron neutrinos and an active-sterile neutrino admixture. We limit our analysis to the allowed LMA region of solar neutrino oscillations. We show in Sec. III how one can use the CC measurements with SNO and KamLAND to determine an accurate total ${ }^{8} \mathrm{~B}$ solar neutrino flux including experimental and theoretical uncertainties and the possibility of an appreciable active-sterile admixture. We switch to the ${ }^{7} \mathrm{Be}$ flux in Sec. IV and evaluate how well one can determine the total ${ }^{7} \mathrm{Be}$ solar neutrino flux by also using the results of the gallium (GALLEX, SAGE, and GNO) solar neutrino experiments or the chlorine experiment. In Sec. V, we investigate how well the total ${ }^{8} \mathrm{~B}$ and ${ }^{7} \mathrm{Be}$ neutrino fluxes can be determined using the combined measurements of KamLAND and $\nu$ - $e$ scattering observed in the Super-Kamiokande (Sec. V A) and BOREXINO (Sec. V B) detectors. We show that even in the presence of active-sterile admixtures the total ${ }^{7} \mathrm{Be}$ solar neutrino flux may be measured with relatively high accuracy by comparing results from the KamLAND and the BOREXINO experiments. We describe and analyze in Sec. VI three strategies for determining the total $p p$ solar neutrino flux in the absence of a dedicated experiment that measures separately the $p p$ neutrinos. We summarize and discuss our principal conclusions in Sec. VII.

We urge the reader to turn first to Sec. VII and read the summary and discussion of our main results and their implications. The rest of the paper can then be understood more easily.

\section{PRESENT KNOWLEDGE OF THE ${ }^{8}$ B NEUTRINO FLUX}

We generalize in this section the determination of the ${ }^{8} \mathrm{~B}$ neutrino flux to the case in which $\nu_{e}$ oscillates into a state that is a linear combination of active $\left(\nu_{a}\right)$ and sterile $\left(\nu_{s}\right)$ neutrino states

$$
\nu_{e} \rightarrow \cos \eta \nu_{x}+\sin \eta \nu_{s}
$$

where $\eta$ is the parameter that describes the active-sterile admixture. This admixture arises naturally in the framework of 4- $\nu$ mixing [28]. The total ${ }^{8} \mathrm{~B}$ neutrino flux can be written

$$
\phi\left({ }^{8} \mathrm{~B}\right)_{\text {total }}=\phi\left(\nu_{e}\right)+\phi\left(\nu_{x}\right)+\phi\left(\nu_{s}\right)
$$

where $\phi\left(\nu_{S}\right)=\tan ^{2} \eta \times \phi\left(\nu_{x}\right)$. Clearly, the larger the sterile component, the larger the value of $\phi\left({ }^{8} \mathrm{~B}\right)_{\text {total }}$ that is inferred from the experimental data.

We have performed a global analysis of the solar neutrino data treating the total ${ }^{8} \mathrm{~B}$ neutrino flux as a free parameter. The details of the analysis procedure are the same as those used in Ref. [22] except where we explicitly state otherwise. We concentrate here on the LMA region $0.1<\tan ^{2} \theta<10$, $10^{-5}<\Delta m^{2} / \mathrm{eV}^{2}<10^{-3}$.

To take account of the possibility of oscillations into sterile neutrinos, we determine the allowed regions in the parameter space defined by $\Delta m^{2}, \tan ^{2} \theta$, and a third parameter, $\cos ^{2} \eta$, that is defined by Eq. (5). It is convenient to introduce the dimensionless parameter

$$
f_{B}=\frac{\phi\left({ }^{8} \mathrm{~B}\right)_{\text {total }}}{\phi\left({ }^{8} \mathrm{~B}\right)_{\mathrm{SSM}}},
$$




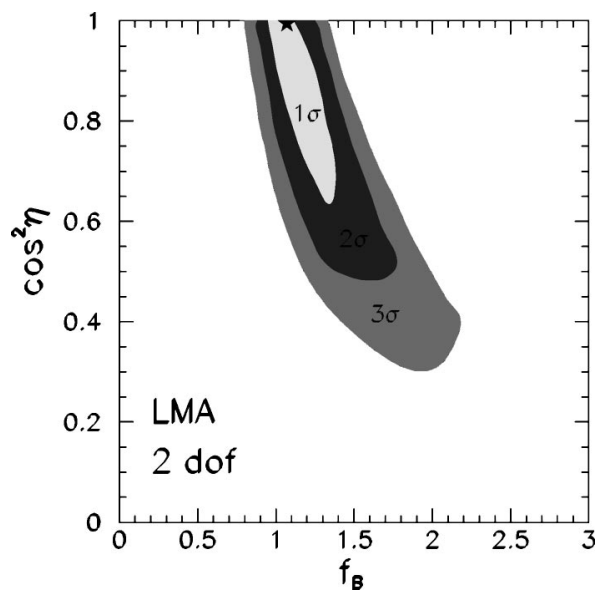

FIG. 2. The dependence of the inferred ${ }^{8} \mathrm{~B}$ flux on the activesterile admixture. The figure shows, as a function of the activesterile admixture, i.e., $\cos ^{2} \eta$, the allowed range of the ${ }^{8} \mathrm{~B}$ solar neutrino flux at $1 \sigma, 2 \sigma$, and $3 \sigma$ C.L. The star indicates the global best fit point for all the currently available solar and reactor data; the star lies at $f_{B}=1.07$ and $\eta=0.0$ (purely active neutrinos).

where $f_{B}$ is the total ${ }^{8} \mathrm{~B}$ neutrino flux in units of the predicted standard solar model flux. The allowed range of $f_{B}$ in the three-dimensional space of neutrino parameters $\Delta m^{2}$, $\tan ^{2} \theta$, and $\cos ^{2} \eta$, is determined by the equation

$$
\chi^{2}\left(f_{B}\right) \leqslant \chi_{\min }^{2}+\Delta \chi^{2}(1, \text { C.L. }) .
$$

Here $\Delta \chi^{2}(1$, C.L. $)$ is the change in $\chi^{2}$ that corresponds to a specified confidence limit (C.L.) for one degree of freedom. The computed values of $\chi^{2}$ are minimized for each value of $f_{B}$ with respect to $\Delta m^{2}, \tan ^{2} \theta$, and $\cos ^{2} \eta$.

Table I shows the currently allowed range for $f_{B}$ for both the more general case where a mixture of active and sterile neutrinos is assumed and for the more conventional case in which only active neutrinos are considered. For purely active neutrinos, the $1 \sigma$ range is

$$
f_{B, \text { active }}=1.07 \pm 0.08
$$

and for an arbitrary mixture of active and sterile neutrinos, the $1 \sigma$ range is

$$
f_{B \text {, active sterile }}=1.07_{-0.08}^{+0.18} \text {. }
$$

The result shown earlier in Eq. (4) for purely active neutrinos is taken from Table I.

How does the possible existence of sterile neutrinos affect the allowed range of ${ }^{8} \mathrm{~B}$ neutrino fluxes? We can calculate the dependence of the allowed range of $f_{B}$ upon $\cos ^{2} \eta$ with the aid of the inequality

$$
\chi^{2}\left(f_{B}, \cos ^{2} \eta\right) \leqslant \chi_{\min }^{2}+\Delta \chi^{2}(2, \text { C.L. }) .
$$

Here $\Delta \chi^{2}(2$, C.L. $)$ is the change in $\chi^{2}$ for a specified C.L. that corresponds to two degrees of freedom ; the computed values of $\chi^{2}$ are minimized at each point with respect to $\Delta m^{2}$ and $\tan ^{2} \theta$.

Figure 2 shows the range of $f_{B}$ as a function of the active- sterile admixture $\cos ^{2} \eta$ that is obtained from Eq. (11) for the $1 \sigma, 2 \sigma$, and $3 \sigma$ allowed regions. The allowed regions are defined respect to the global minimum, which corresponds to purely active oscillations with $\Delta m^{2}=5.0 \times 10^{-5} \mathrm{eV}^{2}, \tan ^{2} \theta$ $=0.42$, and $f_{B}=1.07$.

Although pure sterile oscillations are forbidden at the $3 \sigma$ C.L. (see Fig. 2), a large sterile admixture in the solar oscillations is still allowed. In fact, with the currently available data, the largest allowed value at $3 \sigma$ of the sterile ${ }^{8} \mathrm{~B}$ neutrino flux corresponds to $f_{B}=2.2$ and $\cos ^{2} \eta=0.3$ (for $2 N_{\mathrm{DF}}$ ). For this extreme case, we find

$$
f_{B \text {, sterile } \max }=1.1 \text {. }
$$

The quantity $f_{B \text {,sterile max }}$ that appears in Eq. (12) is defined, analogous to $f_{B}$ in Eq. (7), by the relation $f_{B \text {,sterile max }}$ $=\phi\left({ }^{8} \mathrm{~B}\right)_{\text {sterile } \max } / \phi\left({ }^{8} \mathrm{~B}\right)_{\mathrm{SSM}}$. The maximum value of $f_{B \text {, sterile max }}$ is as large as the sum of the active ${ }^{8} \mathrm{~B}$ neutrino fluxes $\left(f_{x}+f_{e}=1.1\right.$, where $f_{e}=0.348$, see Ref. [4]) for this special case ${ }^{4}$

What is the maximum allowed sterile contamination of the ${ }^{8} \mathrm{~B}$ solar neutrino flux? Minimizing $\chi^{2}$ for the global solution with respect to $\Delta m^{2}, \tan ^{2} \theta$, and $f_{B}$, we find that the allowed range of $\cos ^{2} \eta$ satisfies

$$
0.75(0.40) \leqslant \cos ^{2} \eta \leqslant 1.0
$$

at $1 \sigma($ or $3 \sigma)$.

\section{HOW CAN WE DETERMINE THE TOTAL ${ }^{8}$ B NEUTRINO FLUX USING CC REACTIONS?}

In this section, we will show how one can determine the allowed range of the total ${ }^{8} \mathrm{~B}$ neutrino flux using the results of the KamLAND reactor neutrino experiment and the SNO $\mathrm{CC}$ solar neutrino experiment. We shall also estimate the accuracy with which one can determine the total ${ }^{8} \mathrm{~B}$ flux.

Since we consider here only $\mathrm{CC}$ reactions that result from disappearance experiments, the only difference between the role of active neutrinos $\nu_{\mu}$ and $\nu_{\tau}$ and sterile neutrinos $\nu_{\text {sterile }}$ arises from matter effects in the Earth and in the Sun. Since sterile neutrinos do not interact with matter, the effective potential for the $\nu_{e}-\nu_{s}$ evolution in matter is $V_{e s}=V_{e}-V_{s}$ $=V_{\mathrm{CC}}+V_{\mathrm{NC}}$, since $V_{s}=0$. The effective potential $V_{e s}$ is approximately half the potential for $\nu_{e}-\nu_{a}, V_{e a}=V_{e}-V_{a}$ $=V_{\mathrm{CC}}$, where $V_{a}$ is the potential for the active neutrinos $\nu_{\mu}$ and $\nu_{\tau}$. (The difference is exactly half for a medium with equal number of neutrons, protons, and electrons because $V_{a}=V_{\mathrm{NC}}=-G_{F} N_{n} / \sqrt{2} \sim-V_{\mathrm{CC}} / 2$ with $V_{\mathrm{CC}}=\sqrt{2} G_{F} N_{e}$.) We shall evaluate the expected dependence of the inferred total ${ }^{8} \mathrm{~B}$ flux on the admixture of sterile neutrinos [see Eq. (5)].

In Sec. III A we present the formulas that are used to determine the ${ }^{8} \mathrm{~B}$ flux with the aid of the KamLAND and SNO CC experiments and in Sec. III B we illustrate the ef-

\footnotetext{
${ }^{4}$ For $2 N_{\mathrm{DF}}$, the maximum allowed value of $f_{B}$ is 2.2 at $3 \sigma$, but is 1.7 for $1 N_{\mathrm{DF}}$, see Table I. We have given the maximum value for $1 N_{\mathrm{DF}}$ in the abstract.
} 


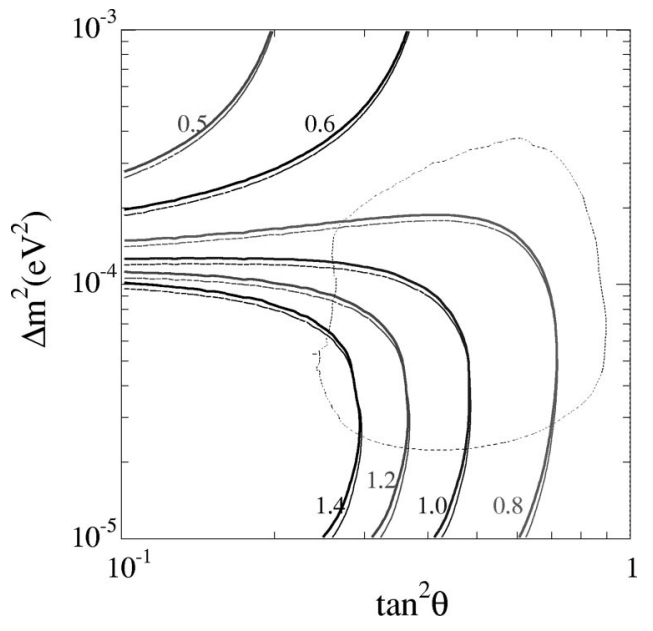

FIG. 3. Isocontours for the total ${ }^{8} \mathrm{~B}$ neutrino flux. The figure compares isocontours for the ${ }^{8} \mathrm{~B}$ flux assuming purely active neutrino oscillations (thicker lines) with the flux that would be inferred for a $75 \%$ active- $25 \%$ sterile admixture (thinner lines). The results refer to a hypothetical comparison of measurements from the KamLAND reactor experiment and the SNO CC experiment. We also show (dotted contour) the $3 \sigma$ allowed region obtained by a global fit to all of the allowed solar and reactor data (see Fig. 1).

fect on the inferred ${ }^{8} \mathrm{~B}$ flux of the maximum allowed (at $1 \sigma$ ) sterile admixture. We estimate in Sec. III C the precision with which the ${ }^{8} \mathrm{~B}$ flux can be determined including all the principal known sources of uncertainties.

The reader who is interested in how well we can determine the ${ }^{8} \mathrm{~B}$ flux, but does not need to know the details of the procedure, can get the main results by glancing at Fig. 2 and Fig. 3 and Table II. In Sec. V A, we investigate how well the total ${ }^{8} \mathrm{~B}$ flux can be determined using KamLAND in combination with the $\nu$-e scattering experiment SuperKamiokande.

TABLE II. Values of $f_{B}$ and associated uncertainties obtainable from the SNO CC and KamLAND experiments. The table presents the best-fit values for $f_{B}$ (the total ${ }^{8} \mathrm{~B}$ neutrino flux divided by the predicted standard solar model ${ }^{8} \mathrm{~B}$ neutrino flux) and associated uncertainties for a representative set of possible oscillation parameters. We have used $\delta\left(f_{B}\right)_{\mathrm{SNO}, \exp }=6.15 \%$ and $\delta\left(f_{B}\right)_{\mathrm{SNO}, \mathrm{CS}}=2 \%$. We consider active-sterile neutrino admixtures permitted by the currently allowed global oscillation solution [see Eq. (12)].

\begin{tabular}{|c|c|c|c|c|}
\hline$\Delta \bar{m}^{2}$ & $\tan ^{2} \bar{\theta}$ & $f_{B}$ & $\sigma\left(\left\langle P_{e e}\right\rangle_{\mathrm{SNO}, \text { KamLAND }}\right)$ & $\begin{array}{c}\text { Total } \\
\%\end{array}$ \\
\hline $5.0 \times 10^{-5}$ & $4.2 \times 10^{-1}$ & 1.09 & $\begin{array}{l}+7.4 \\
-6.9\end{array}$ & $\begin{array}{l}+8.4 \\
-8.1\end{array}$ \\
\hline $5.0 \times 10^{-5}$ & $5.01 \times 10^{-1}$ & 0.98 & $\begin{array}{l}-0.9 \\
+7.2 \\
-64\end{array}$ & $\begin{array}{l}-8.1 \\
+8.3 \\
-78\end{array}$ \\
\hline $5.0 \times 10^{-5}$ & $2.51 \times 10^{-1}$ & 1.51 & $\begin{array}{l}+8.5 \\
+8.5 \\
-85\end{array}$ & $\begin{array}{l}+9.0 \\
+8.1\end{array}$ \\
\hline $7.94 \times 10^{-5}$ & $4.2 \times 10^{-1}$ & 1.02 & $\begin{array}{l}-8.5 \\
+6.6 \\
-7.3\end{array}$ & $\begin{array}{l}-8.9 \\
+9.0 \\
-9.6\end{array}$ \\
\hline $7.94 \times 10^{-5}$ & $5.01 \times 10^{-1}$ & 0.94 & $\begin{array}{r}+6.9 \\
+-92\end{array}$ & $\begin{array}{l}+9.0 \\
-9.2\end{array}$ \\
\hline $7.94 \times 10^{-5}$ & $2.51 \times 10^{-1}$ & 1.30 & $\begin{array}{l}+7.1 \\
-7.9\end{array}$ & $\begin{array}{l}+9.4 \\
-10\end{array}$ \\
\hline $3.16 \times 10^{-5}$ & $4.2 \times 10^{-1}$ & 1.01 & $\begin{array}{l}+4.3 \\
-53\end{array}$ & $\begin{array}{l}+7.5 \\
+-8.5\end{array}$ \\
\hline $3.16 \times 10^{-5}$ & $5.01 \times 10^{-1}$ & 0.98 & $\begin{array}{l}+6.1 \\
+5.1\end{array}$ & $\begin{array}{l}-8.2 \\
+8.7 \\
-8.0\end{array}$ \\
\hline $3.16 \times 10^{-5}$ & $2.51 \times 10^{-1}$ & 1.57 & $\begin{array}{r}+6.9 \\
+8.1\end{array}$ & $\begin{array}{l}0.0 \\
+9.2 \\
-10\end{array}$ \\
\hline
\end{tabular}

\section{A. Relations that determine the total flux}

Suppose KamLAND observes a signal that corresponds to LMA $\bar{\nu}_{e}$ oscillations with parameters $\left(\Delta \bar{m}^{2}, \tan \bar{\theta}^{2}\right)$. We assume the validity of the CPT theorem so that constraints on antineutrino oscillation parameters obtained from the KamLAND experiment apply to solar neutrino experiments. We can then extract the ${ }^{8} \mathrm{~B}$ neutrino flux from the following relation:

$$
f_{B}=\frac{R_{\mathrm{SNO}}^{\mathrm{CC}, \exp }}{R_{\mathrm{SNO}}^{\mathrm{SSM}}} \times \frac{1}{\left\langle P_{e e}\left(\Delta m^{2}, \tan ^{2} \theta\right)\right\rangle_{\mathrm{SNO}}},
$$

where

$$
R_{\mathrm{SNO}}^{\mathrm{SSM}}=\int d E_{\nu} \phi^{\mathrm{SSM}}\left({ }^{8} \mathrm{~B}, E_{\nu}\right) \sigma_{e}\left(E_{\nu}\right)=2.87 R_{\mathrm{SNO}}^{\mathrm{CC}, \exp }
$$

is the $\mathrm{CC}$ rate for the SNO experiment [4] that is predicted $[11,22]$ by the standard solar model in the absence of oscillations and $\left\langle P_{e e}\left(\Delta m^{2}, \tan ^{2} \theta\right)\right\rangle_{\mathrm{SNO}}$ is the average survival probability for electron-flavor neutrinos created in the Sun. Also, $E_{\nu}$ is the neutrino energy and $\sigma_{e}$ is the weighted average $\nu_{e}$-d interaction cross-section, including the experimental energy resolution function $\operatorname{Res}\left(T, T^{\prime}\right)$, where $T\left(T^{\prime}\right)$ is the measured (true) recoil kinetic energy of the electron. Thus

$$
\sigma_{e}\left(E_{\nu}\right)=\int_{T_{\min }}^{T_{\max }} d T \int_{0}^{T_{\max }{ }^{\prime}\left(E_{\nu}\right)} d T^{\prime} \operatorname{Res}\left(T, T^{\prime}\right) \frac{d \sigma_{e}\left(E_{\nu}, T^{\prime}\right)}{d T^{\prime}} .
$$

The lower limit $T_{\min }$ in the integral in Eq. (16) is taken here to be the threshold used by the SNO Collaboration in Ref. [4] $\left(T_{\min }=5 \mathrm{MeV}\right)$. The calculated value for the CC rate is not sensitive to the assumed value of $T_{\max }$, as long as $T_{\max }$ $\geqslant 17 \mathrm{MeV}$.

The energy-averaged survival probability $\left\langle P_{e e}\left(\Delta m^{2}, \tan ^{2} \theta\right)\right\rangle_{\mathrm{SNO}}$ for $\nu_{e}$ at SNO can be computed using the propagation parameters $\left(\Delta \bar{m}^{2}, \tan ^{2} \bar{\theta}\right)$ observed at KamLAND. Thus

$$
\begin{aligned}
& \left\langle P_{e e}\left(\Delta m^{2}, \tan ^{2} \theta\right)\right\rangle_{\mathrm{SNO}} \\
& =\frac{\int d E_{\nu} \phi^{\mathrm{SSM}}\left({ }^{8} \mathrm{~B}, E_{\nu}\right) \sigma_{e}\left(E_{\nu}\right) P_{e e}\left(E_{\nu}, \Delta \bar{m}^{2}, \tan ^{2} \bar{\theta}\right)}{R_{\mathrm{SNO}}^{\mathrm{SSM}}} .
\end{aligned}
$$

\section{B. Illustrative dependence of total flux upon active-sterile admixture}

How much does a sterile neutrino admixture affect the inferred total ${ }^{8} \mathrm{~B}$ neutrino flux? The dominant dependence on the sterile admixture arises from matter effects within the Sun for larger $\Delta m^{2}$ and within the Earth for smaller $\Delta m^{2}$.

Figure 3 shows the isocontours of $f_{B}$ in the LMA region for the pure active case (thicker lines) and for the activesterile case $\cos ^{2} \eta=0.75$ (thinner lines). The isocontours are 
not very different for the two cases. Moreover, the dependence on Earth matter effects can be avoided experimentally by using only the daytime $\mathrm{CC}$ measurement, once sufficient statistics are available. The robustness of the inferred ${ }^{8} \mathrm{~B}$ solar neutrino flux can be tested by comparing the ${ }^{8} \mathrm{~B}$ flux inferred using only daytime $\mathrm{CC}$ measurements with the flux that is inferred when nighttime data (with corrections for Earth matter effects) are added to the daytime data.

In Fig. 3, we also show the $3 \sigma$ LMA contour (the dotted contour) obtained by the global analysis of the solar neutrino data for the purely active case. Within the $3 \sigma$ LMA region, the maximum difference between the value of $f_{B}$ inferred allowing for possible sterile neutrinos and the value obtained assuming only active neutrino oscillations is +0.9 and $-3.5 \%$. The dependence upon the active-sterile mixture could become negligible if the correct $\Delta m^{2}$ lies in the lower part of the LMA region and only daytime data is used from the SNO CC measurements. We conclude from Fig. 3 that existence of sterile neutrinos will not prevent an accurate measurement of the total ${ }^{8} \mathrm{~B}$ neutrino flux.

\section{How accurately can the total ${ }^{8} \mathrm{~B}$ flux be determined?}

What is the overall precision expected in the determination of the total ${ }^{8} \mathrm{~B}$ flux? From Eq. (14) we can derive the anticipated precision as

$$
\begin{aligned}
\left(\frac{\sigma\left(f_{B}\right)}{f_{B}}\right)^{2}= & \left(\frac{\sigma\left(R_{\mathrm{SNO}}^{\mathrm{CC}, \mathrm{exp}}\right)}{R_{\mathrm{SNO}}^{\mathrm{CC}, \mathrm{exp}}}\right)^{2}+\left(\frac{\sigma\left(R_{\mathrm{SNO}}^{\mathrm{SSM}}\right)}{R_{\mathrm{SNO}}^{\mathrm{SSM}}}\right)^{2} \\
+ & \left.\left(\frac{\sigma\left(\left\langle P_{e e}\right\rangle_{\mathrm{SNO}, \mathrm{KamLAND}}\right)}{\left\langle P_{e e}\right\rangle_{\mathrm{SNO}, \mathrm{KamLAND}}}\right)\right)^{2} \\
\equiv & \delta\left(f_{B}\right)_{\mathrm{SNO}, \mathrm{exp}}^{2}+\delta\left(f_{B}\right)_{\mathrm{SNO}, \text { cross section }}^{2} \\
& +\delta\left(f_{B}\right)_{\mathrm{SNO}, \mathrm{KamLAND}}^{2}
\end{aligned}
$$

where the $1 \sigma$ errors are combined quadratically.

The current value of the first term in Eq. (18), the $1 \sigma$ uncertainty (statistical and systematic) of the measured CC rate in $\mathrm{SNO}$, is [1] $\delta\left(f_{B}\right)_{\mathrm{SNO}, \exp }=6.15 \%$. This uncertainty will undoubtedly decrease as the results of analyzing more SNO CC data are reported.

The second term in Eq. (18) represents the uncertainty in the $\nu_{e^{-}}{ }^{2} \mathrm{H}$ absorption cross section. Much progress has been made recently in evaluating this cross section, see, e.g., Refs. $[41,42]$, which has led to an estimate $\sim 1 \%$ for the cross section uncertainties other than radiative corrections. No definitive calculation has yet been made of the radiative correction for the CC reaction, but a reasonable estimate [41] is that the cross sections given in Ref. [41] might be increased by $2 \%$. We adopt here a conservative uncertainty of $\delta\left(f_{B}\right)_{\mathrm{SNO}, \text { c.s. }}=2 \%$; the precise value chosen for $\delta\left(f_{B}\right)_{\mathrm{SNO}, \text { c.s. }}$ is not very important at this stage since other uncertainties are dominant. The hep neutrino flux contribution to $\Delta f_{B}$ is negligible for our purposes [4,5,11].

A detailed simulation is required to estimate $\sigma\left(\left\langle P_{e e}\right\rangle_{\mathrm{SNO}, \mathrm{KamLAND}}\right)$, i.e., the uncertainty in the average electron neutrino survival probability for ${ }^{8} \mathrm{~B}$ solar neutrinos observed in the SNO CC experiment as will be determined by future KamLAND measurements. Here is how we estimate this uncertainty. We generate the expected KamLAND signal for a fine grid of points $\left(\Delta \bar{m}^{2}, \tan ^{2} \bar{\theta}\right)$ that spans the space of the allowed oscillation parameters determined from solar neutrino experiments. For each grid point, we obtain the allowed region by a $\chi^{2}$ analysis. We use statistical errors corresponding to three years of data taking at KamLAND, observing antineutrinos from reactors working at a constant $78 \%$ of the maximal power. To be conservative, we also assumed a neutrino energy threshold of $3.5 \mathrm{MeV}$, in order to ensure that the effects of natural radioactivity would be small. More details on the KamLAND experiment can be found in Ref. [31]; details regarding the neutrino cross sections, statistical procedures, and reactor fluxes used in the present paper are described in Refs. [38,39].

In computing the inferred values of $f_{B}$, we take account of the fact that there could be a significant component of sterile neutrinos in the incident ${ }^{8} \mathrm{~B}$ solar neutrino flux. We therefore consider all active-sterile admixtures permitted by the global oscillation solution shown in Fig. 1. The numerical constraint on the currently allowed admixture is given in Eq. (13).

In principle, for each simulated point there are two allowed KamLAND regions, one around $\Delta \bar{m}^{2}$ and $\tan ^{2} \bar{\theta}$ and another around $\Delta \bar{m}^{2}$ and $\tan ^{2}(\pi / 2-\bar{\theta})$. We discuss here only the range of parameters within the first octant for the mixing angle since global solar neutrino solutions show $[6-8,20-22,32]$ that the first octant is preferred. For each simulated KamLAND allowed region centered on a specific $\left(\Delta \bar{m}^{2}, \tan ^{2} \bar{\theta}\right)$, we compute $\sigma\left(\left\langle P_{e e}\right\rangle_{\text {KamLAND }}\right)$. We repeated this procedure for a grid of $81 \times 41$ points in the range 0.1 $<\tan ^{2} \bar{\theta}<10,10^{-5}<\Delta \bar{m}^{2}<1 \times 10^{-3}$. The estimated uncertainty in $\left\langle P_{e e}\right\rangle_{\mathrm{SNO}}$ KamLAND varies with the grid point $\left(\Delta \bar{m}^{2}, \tan ^{2} \bar{\theta}\right)$.

Table II presents the best-fit value of $f_{B}$, the uncertainty in $\sigma\left(\left\langle P_{e e}\right\rangle_{\mathrm{SNO}, \mathrm{KamLAND}}\right)$, and the total expected uncertainty in inferring $f_{B}$ from the combined SNO CC and the KamLAND neutrino reactor measurements for a representative set of possible results for $\Delta \bar{m}^{2}$ and $\tan ^{2} \bar{\theta}$. In all the cases shown in Table II, we have used $\delta\left(f_{B}\right)_{\mathrm{SNO} \text {,exp }}=6.15 \%$ and $\delta\left(f_{B}\right)_{\text {SNO,CS }}=2 \%$ (see previous discussion). The uncertainty contributed by the possibility that sterile neutrinos exist, i.e., $\eta \neq 0$, is rather modest; $\sigma\left(\left\langle P_{e e}\right\rangle_{\mathrm{SNO}, \mathrm{KamLAND}}\right)$ is typically reduced by $\sim 1 \%$ from the value shown in Table II.

Figure 4 shows the 9 and $14 \%$ contours for the maximum percentage deviation from the best-fit $f_{B}$ value. To provide a context, the figure also displays the $1 \sigma, 2 \sigma$, and $3 \sigma$ allowed LMA regions obtained by a global fit to the available solar and reactor data. Within almost all of the current $1 \sigma$ LMA allowed region, the comparison of the KamLAND and the SNO CC data will determine the total ${ }^{8} \mathrm{~B}$ flux with an uncertainty that is less than $14 \%$; the uncertainty can be less than $10 \%$ over a significant fraction of the current $1 \sigma$ allowed domain. About $\sim 6 \%$ of the current estimated uncertainty is due to the experimental error in the SNO CC measurement, which hopefully will be reduced as more CC data are accumulated. 


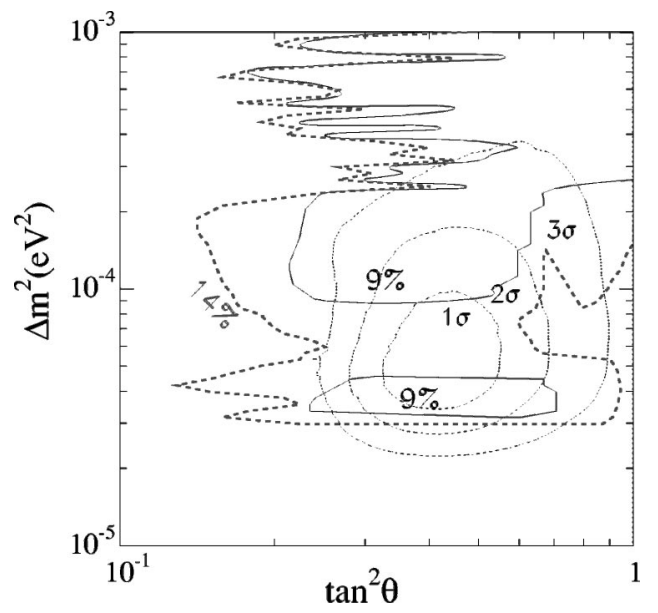

FIG. 4. Accuracy of determining the total ${ }^{8} \mathrm{~B}$ neutrino flux. The figure displays $1 \sigma$ contours for the percentage accuracy in determining the ${ }^{8} \mathrm{~B}$ flux that can be obtained from the combined SNO $\mathrm{CC}$ and KamLAND data. The uncertainties were calculated from Eq. (18) and the currently allowed regions for the neutrino oscillation parameters were obtained by a global fit to all of the allowed solar and reactor data (see Fig. 1).

For some purposes, it is convenient to know what is the average expected accuracy in the determination of $f_{B}$. We have computed this average with the aid of a Monte Carlo sampling of the currently allowed solar neutrino oscillation parameters shown in Fig. 1. The average positive and negative uncertainties are approximately equal (see Table II). We find an average $1 \sigma$ uncertainty of

$$
\sigma\left(f_{B}\right)=9.6 \% \text {. }
$$

A very significant component of $\sigma\left(f_{B}\right)$ comes from $\sigma\left(\left\langle P_{e e}\right\rangle_{\mathrm{SNO}}\right.$ KamLAND $)$. We find, averaged over the current $1 \sigma$ solar neutrino oscillation region

$$
\sigma\left(\left\langle P_{e e}\right\rangle_{\mathrm{SNO}, \mathrm{KamLAND}}\right)=7 \% .
$$

The sterile neutrino contribution to the ${ }^{8} \mathrm{~B}$ neutrino flux can be determined by subtracting the active neutrino flux from the total neutrino flux. Thus,

$$
f_{B, \text { sterile }}=\frac{R_{\mathrm{SNO}}^{\mathrm{CC}, \exp }-R_{\mathrm{SNO}}^{\mathrm{NC}, \exp }\left\langle P_{e e}\left(\Delta m^{2}, \tan ^{2} \theta\right)\right\rangle_{\mathrm{SNO}}}{R_{\mathrm{SNO}}^{\mathrm{SSM}}\left\langle P_{e e}\left(\Delta m^{2}, \tan ^{2} \theta\right)\right\rangle_{\mathrm{SNO}}} .
$$

Using, as described above, the $1 \sigma$ errors for the total and the active fluxes of 9.7 and $8 \%$ (assumed for the SNO NC measurement), respectively, we estimate that the sterile component of the ${ }^{8} \mathrm{~B}$ neutrino flux can be determined to a precision of about $12.5 \%$.

\section{HOW WELL CAN WE DETERMINE THE TOTAL ${ }^{7}$ Be FLUX USING CC (RADIOCHEMICAL) EXPERIMENTS?}

We show in this section how the total ${ }^{7} \mathrm{Be}$ solar neutrino flux can be determined, with the judicial aid of other neutrino fluxes predicted by the standard solar model [11], by combining the results of the GALLEX [15],GNO [16], and
SAGE [14] gallium solar neutrino experiments with the KamLAND and SNO CC measurements. We shall also explore the extent to which the chlorine experiment $[12,43]$ can provide independent information about the ${ }^{7} \mathrm{Be}$ solar neutrino flux.

We limit ourselves in this section to detectors that only observe $\nu_{e}$ or $\bar{\nu}_{e}$, specifically, we consider here only the radiochemical gallium and chlorine experiments and the reactor antineutrino experiment KamLAND. This limitation simplifies the calculations with respect to the role of the sterile neutrinos. However, the radiochemical experiments suffer from the disadvantage of a lack of energy discrimination, which introduces uncertainties involving the roles of the $p p$, pep, and $\mathrm{CNO}$ neutrinos.

We begin by describing in Sec. IV A the general procedure for determining the ${ }^{7} \mathrm{Be}$ neutrino flux. We then evaluate in Sec. IV B the principal sources of error, taking account of experimental and theoretical uncertainties as well as the possibility of an appreciable sterile neutrino component in the incident solar neutrino flux. We present in Sec. IV C the numerical results for the uncertainties due to different factors and evaluate the overall accuracy with which the total ${ }^{7} \mathrm{Be}$ flux can be determined.

Using data from either the gallium or the chlorine [12] experiments, the same procedure can be applied for inferring the ${ }^{7} \mathrm{Be}$ neutrino flux. For simplicity, we describe the procedure in Secs. IV A-IV C with reference to the more promising case provided by the gallium experiments. In Sec. IV D, we investigate how accurately one can determine the ${ }^{7} \mathrm{Be}$ flux using data from the chlorine experiment instead of the gallium experiment. In Sec. V B, we use the techniques developed in this section to explore the accuracy with which KamLAND and BOREXINO can determine $f_{\mathrm{Be}}$.

\section{A. Procedure for determining the total ${ }^{7}$ Be solar neutrino flux}

Table III shows the neutrino fluxes and the event rates in the gallium solar neutrino experiments that are predicted by the standard solar model $[11,22]$. The table also shows the event rate predicted by the best-fit LMA solution. From Table III it is clear that one must make a strong assumption about the $p p$ neutrino flux in order to determine the ${ }^{7} \mathrm{Be}$ flux. One must also make assumptions regarding the bestvalue and the uncertainties in the $\mathrm{CNO}$ and pep fluxes. This situation is different than the purely empirical procedure described in Sec. III for determining the ${ }^{8} \mathrm{~B}$ neutrino flux; the ${ }^{8} \mathrm{~B}$ solar neutrino flux can be determined independent of all considerations regarding the standard solar model.

We assume throughout this section the correctness of the calculated standard solar model [11] values for the neutrino fluxes and their uncertainties, except for the ${ }^{7} \mathrm{Be}$ and ${ }^{8} \mathrm{~B}$ fluxes which we want to determine from solar neutrino experiments. ${ }^{5}$

\footnotetext{
${ }^{5}$ The ultimate astronomical goal of solar neutrino experiments is to determine all of the solar neutrino fluxes directly from experiment, but there are too few experimental constraints to make this possible at the present time.
} 
TABLE III. Gallium neutrino capture rates and solar neutrino fluxes. The table presents the predicted standard solar model [11] neutrino fluxes and the calculated gallium neutrino capture rates, with $1 \sigma$ uncertainties from all sources (combined quadratically). The neutrino fluxes are the same as in the original BP00 model [11]. The last column of the table presents the capture rate for gallium predicted by the best fit LMA solution. The total rates were calculated using the neutrino absorption cross sections and their uncertainties that are given in Ref. [44].

\begin{tabular}{lccc}
\hline \hline Source & $\begin{array}{c}\text { Flux } \\
\left(10^{10} \mathrm{~cm}^{-2} \mathrm{~s}^{-1}\right)\end{array}$ & $\begin{array}{c}\text { Ga (SSM) } \\
(\mathrm{SNU})\end{array}$ & $\begin{array}{c}\text { Ga (LMA) } \\
(\mathrm{SNU})\end{array}$ \\
\hline$p p$ & $5.95 \times 10^{0}\left(1.00_{-0.01}^{+0.01}\right)$ & 69.7 & 40.4 \\
pep & $1.40 \times 10^{-2}\left(1.00_{-0.015}^{+0.015}\right)$ & 2.8 & 1.51 \\
hep & $9.3 \times 10^{-7}$ & 0.1 & 0.023 \\
${ }^{7} \mathrm{Be}$ & $4.77 \times 10^{-1}\left(1.00_{-0.10}^{+0.10}\right)$ & 34.2 & 18.6 \\
${ }^{8} \mathrm{~B}$ & $5.05 \times 10^{-4}\left(1.00_{-0.16}^{+0.10}\right)$ & 12.2 & 4.35 \\
${ }^{13} \mathrm{~N}$ & $5.48 \times 10^{-2}\left(1.00_{-0.17}^{+0.17}\right)$ & 3.4 & 1.79 \\
${ }^{15} \mathrm{O}$ & $4.80 \times 10^{-2}\left(1.00_{-0.19}^{+0.25}\right)$ & 5.5 & 2.83 \\
${ }^{17} \mathrm{~F}$ & $5.63 \times 10^{-4}\left(1.00_{-0.25}^{+0.25}\right)$ & 0.1 & 0.03 \\
Total & & $128_{-7}^{+9}$ & 69.6 \\
\hline \hline
\end{tabular}

Fortunately, as we shall see, if we assume the SSM predictions and their uncertainties for all but the ${ }^{7} \mathrm{Be}$ and ${ }^{8} \mathrm{~B}$ fluxes, then we can infer an interesting range for the total solar ${ }^{7} \mathrm{Be}$ neutrino flux if we use data from the gallium experiments. The situation is less promising if we use data from the chlorine experiment rather than the gallium experiments.

Suppose that KamLAND observes a signal that corresponds to $\bar{\nu}_{e}$ oscillations with parameters $\left(\Delta \bar{m}^{2}, \tan \bar{\theta}^{2}\right)_{\text {KamLAND }}$, then the expected event rate in the gallium experiments is a sum of the contributions from the different neutrino fluxes, namely,

$$
\begin{aligned}
R_{\mathrm{Ga}}= & f_{B} R_{\mathrm{Ga}}^{{ }^{8} \mathrm{~B}, \mathrm{SSM}}\left\langle P_{e e}\left(\Delta \bar{m}^{2}, \tan ^{2} \bar{\theta}\right)_{\mathrm{KamLAND}}\right\rangle_{\mathrm{Ga}}^{{ }^{8} \mathrm{~B}} \\
& +f_{\mathrm{Be}} R_{\mathrm{Ga}}^{{ }^{7} \mathrm{Be}, \mathrm{SSM}}\left\langle P_{e e}\left(\Delta \bar{m}^{2}, \tan ^{2} \bar{\theta}\right)_{\mathrm{KamLAND}}\right\rangle_{\mathrm{Ga}} \\
& +\sum_{i} f_{i} R_{\mathrm{Ga}}^{\phi_{i}, S S M}\left\langle P_{e e}\left(\Delta \bar{m}^{2}, \tan ^{2} \bar{\theta}\right)_{\mathrm{KamLAND}}\right\rangle_{\mathrm{Ga}}^{\phi_{i}}
\end{aligned}
$$

In the last term in Eq. (22), we include the contributions from hep, pep, $\mathrm{CNO}$, and $p p$ neutrinos. By analogy with Eq. (7), we have defined the factors $f_{i}$ as the ratios between the "true" solar neutrino fluxes and the fluxes predicted by the standard solar model.

We can solve Eq. (22) for the ${ }^{7} \mathrm{Be}$ solar neutrino flux as follows. We substitute into Eq. (22) the value of $f_{B}$ determined, independent of the solar model, from the KamLAND and SNO CC measurements, as discussed in the previous section (Sec. III). We also assume as a first approximation that all the solar neutrino fluxes but the ${ }^{8} \mathrm{~B}$ and ${ }^{7} \mathrm{Be}$ fluxes are equal to the values predicted by the SSM; we investigate later the accuracy of this approximation. With these assumptions, we can then solve for $f_{\mathrm{Be}}$ by equating $R_{\mathrm{Ga}}=R_{\mathrm{Ga}}^{\exp }$ $=72.4 \pm 4.7$ SNU. Thus

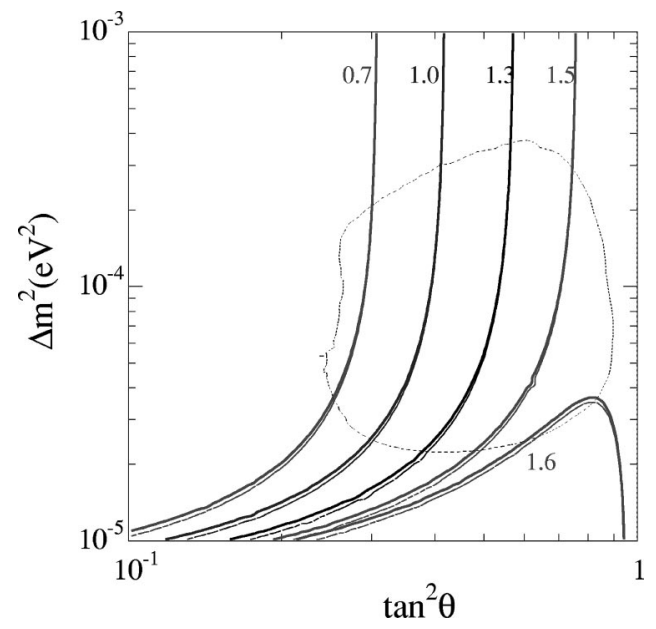

FIG. 5. Isocontours for the total ${ }^{7} \mathrm{Be}$ flux. The figure compares isocontours for the total ${ }^{7} \mathrm{Be}$ flux assuming oscillations between $\nu_{e}$ and purely active neutrinos (thicker lines) or oscillations between $\nu_{e}$ and a $75 \%$ active-25\% sterile admixture (thinner lines). The results were obtained by solving Eq. (23) as described in the text. The differences between the pure active contours and the 75-25\% admixtures are less than $2 \%$ within the currently allowed $3 \sigma$ solution space, which is shown by the dotted line in the figure (see Fig. 1 ). The dotted curve represents the $3 \sigma$ allowed regions from the analysis of the solar data.

$$
\begin{aligned}
& f_{\mathrm{Be}}=\frac{1}{R_{\mathrm{Ga}}^{{ }^{7} \mathrm{Be}, \mathrm{SSM}}\left\langle P_{e e}\left(\Delta \bar{m}^{2}, \tan ^{2} \bar{\theta}\right)_{\mathrm{KamLAND}}\right\rangle_{\mathrm{Ga}}} \\
& \times\left[R_{\mathrm{Ga}}^{\exp }-\sum_{i} R_{\mathrm{Ga}}^{\phi_{i}, \mathrm{SSM}}\left\langle P_{e e}\left(\Delta \bar{m}^{2}, \tan ^{2} \bar{\theta}\right)_{\mathrm{KamLAND}}\right\rangle_{\mathrm{Ga}}^{\phi_{i}}\right. \\
& \left.-R_{\mathrm{Ga}}^{{ }^{8} \mathrm{~B}, \mathrm{SSM}} \frac{R_{\mathrm{SNO}}^{\mathrm{CC}, \exp }}{R_{\mathrm{SNO}}^{\mathrm{CC}, \mathrm{SSM}}} \frac{\left\langle P_{e e}\left(\Delta \bar{m}^{2}, \tan ^{2} \bar{\theta}\right)_{\mathrm{KamLAND}}\right\rangle_{\mathrm{Ga}}{ }^{8 \mathrm{~B}}}{\left\langle P_{e e}\left(\Delta \bar{m}^{2}, \tan ^{2} \bar{\theta}\right)_{\mathrm{KamLAND}}\right\rangle_{\mathrm{SNO}}}\right]
\end{aligned}
$$

Figure 5 shows that for the ${ }^{7} \mathrm{Be}$ neutrino flux the uncertainty due to the potential effect of sterile neutrinos is small. The figure shows the isocontours of $f_{\mathrm{Be}}$ in the LMA region for the case of purely active case (thicker lines) and for the mixture of active and sterile neutrinos with $\cos ^{2} \eta=0.75$ (thinner lines). Within the $1 \sigma(3 \sigma)$ parameter region, the difference in the value of $f_{B}$ between the two oscillation scenarios is less than $1 \%(2 \%)$.

\section{B. Principal sources of uncertainty in determining the ${ }^{7} \mathrm{Be}$ total flux}

The uncertainty in the inferred total ${ }^{7} \mathrm{Be}$ solar neutrino flux can be estimated from Eq. (23). Including just the largest contributions, we can write the fractional uncertainty in the total ${ }^{7} \mathrm{Be}$ neutrino flux as 


$$
\begin{aligned}
\left(\frac{\sigma\left(f_{\mathrm{Be}}\right)}{f_{\mathrm{Be}}}\right)^{2}= & \delta\left(f_{\mathrm{Be}}\right)_{\mathrm{Ga}, \exp }^{2}+\delta\left(f_{\mathrm{Be}}\right)_{\mathrm{Ga}, \text { crosssection }}^{2} \\
& +\delta\left(f_{\mathrm{Be}}\right)_{\mathrm{KamLAND}}^{2}+\delta\left(f_{\mathrm{Be}}\right)_{\mathrm{CNO}}^{2}+\text { smaller terms }
\end{aligned}
$$

where $\delta\left(f_{\mathrm{Be}}\right)_{\mathrm{Ga}, \exp }$ is the uncertainty from the experimental error of the gallium rate, $\delta\left(f_{\mathrm{Be}}\right)_{\mathrm{Ga} \text {, cross section }}$ is the theoretical uncertainty from the calculated $\nu_{e}$-Ga absorption cross sections [44], $\delta\left(f_{\mathrm{Be}}\right)_{\mathrm{KamLAND}}^{2}$ is the uncertainty arising from the allowed range of neutrino parameters determined by KamLAND and SNO [which affects all of the averaged survival probabilities in Eq. (23)], and $\delta\left(f_{\mathrm{Be}}\right)_{\mathrm{CNO}}^{2}$ is the uncertainty due to the quoted errors in the standard solar model calculation of the CNO fluxes (see Table III and Ref. [11]). We have omitted from Eq. (24) a number of sources of error that contribute only relatively small uncertainties. The omitted sources of error [and their range of contributed uncertainties for a representative set of oscillation parameters] include the experimental error in the SNO CC measurements [ 1.2 $\pm 0.6) \%$, the uncertainty in the theoretical absorption cross section for the SNO CC experiment $[(0.7 \pm 0.2) \%]$, and the uncertainty in standard solar model calculation of the $p p$ neutrino flux $[(0.4 \pm 0.1) \%]$.

We now discuss how we estimate the uncertainties in $f_{\mathrm{Be}}$. Because they require special treatment, we first discuss the uncertainty arising from the theoretical neutrino capture cross sections for gallium and then discuss the uncertainty resulting from the range of allowed neutrino parameters determined by KamLAND. ${ }^{6}$ To evaluate the uncertainties arising from the gallium absorption cross sections for neutrino sources with continuous energy distributions, we use Tables 2-4 of Ref. [44]; these tables give the best-estimate and the $\pm 3 \sigma$ limits for the theoretical cross sections. For the neutrino lines from ${ }^{7} \mathrm{Be}$ and $p e p$, we have checked that the shape of the line [45] does not affect significantly the error estimate. Therefore, we use for the ${ }^{7} \mathrm{Be}$ and pep lines the error estimates given in Eq. (41) and Eq. (42) of Ref. [44]. We have adopted the conservative procedure described in Sec. XII A 4 of Ref. [44], in which the uncertainties in all of the low energy $(<2 \mathrm{MeV})$ cross sections are fully correlated, while the uncertainties for the $\left({ }^{8} \mathrm{~B}\right)$ neutrinos above $2 \mathrm{MeV}$ are treated separately. All of the cross sections for low energy neutrinos move up or down together, reflecting the fact that the dominant uncertainties for low energy neutrinos are common to all sources. For higher-energy neutrinos, a number of excited states dominate the calculated absorption cross section (For a more explicit description of how the cross section errors are treated, see the Appendix.)

To calculate the uncertainty associated with the range of allowed neutrino parameters determined by KamLAND, we first solve Eq. (23) with the appropriate average survival probabilities computed for each neutrino oscillation point

\footnotetext{
${ }^{6}$ The SNO CC results are used to select the first quadrant for $\theta$ (see Fig. 1), but for brevity we refer to the range of neutrino parameters determined by KamLAND.
}

$\left(\Delta \bar{m}^{2}, \tan ^{2} \bar{\theta}\right)$ in the allowed region (see Fig. 1). We consider active-sterile neutrino admixtures permitted by the currently allowed global oscillation solution [see Eq. (13)]. The solution of Eq. (23) determines $f_{\mathrm{Be}}$ (best fit) for that particular point in oscillation parameter space. Then we construct a $1 \sigma$ allowed region, a set of points $\left(\Delta m^{2}, \tan ^{2} \theta\right)$, around the chosen point using the simulated characteristics of the KamLAND experiment (see Sec. III C and Refs. [31,38,39]). We define $\delta\left(f_{\mathrm{Be}}\right)_{\text {KamLAND }}$ for the chosen $\left(\Delta \bar{m}^{2}, \tan ^{2} \bar{\theta}\right)$ to be the maximum (or minimum) value of $\left[f_{\mathrm{Be}}\left(\Delta m^{2}, \tan ^{2} \theta\right)\right.$ $-f_{\mathrm{Be}}$ (best fit) $] / f_{\mathrm{Be}}$ (best fit). In practice, the inclusion of sterile neutrinos only slightly affects the computed range of $\delta\left(f_{\mathrm{Be}}\right)_{\text {KamLAND }}$.

For all other quantities, we estimate the associated uncertainty at a particular point $\left(\Delta \bar{m}^{2}, \tan ^{2} \bar{\theta}\right)$ in the following way. Given a source of uncertainty $i$ (for example, the measured capture rate for the gallium experiments) with $1 \sigma$ error $\sigma_{i}$, we obtain $\delta\left(f_{\mathrm{Be}}\right)_{i}$ from the relation

$$
\delta\left(f_{\mathrm{Be}}\right)_{i}=\frac{f_{\mathrm{Be}}\left(i \pm \sigma_{i}\right)-f_{\mathrm{Be}}(i)}{f_{\mathrm{Be}}(i)} .
$$

Here we denote by $f_{\mathrm{Be}}(i)$ the value of $f_{\mathrm{Be}}$ obtained from Eq. (23) when all the parameters are assigned their best-estimate values. The quantity $f_{\mathrm{Be}}\left(i \pm \sigma_{i}\right)$ is calculated from Eq. (23) using the best-estimate values of all variables except $i$; the variable $i$ is set equal to its best-fit value \pm the corresponding $1 \sigma$ uncertainty. In calculating $\delta\left(f_{\mathrm{Be}}\right)_{\mathrm{CNO}}^{2}$, we shift the three CNO neutrino fluxes by $\pm 1 \sigma$ simultaneously and in the same direction, reflecting the correlation between the CNO fluxes in the standard solar model.

The uncertainty $\delta\left(f_{\mathrm{Be}}\right)_{i}$ that is calculated from Eq. (25) will in general depend upon the assumed value for $\left(\Delta \bar{m}^{2}, \tan ^{2} \bar{\theta}\right)$ within the KamLAND allowed region. This dependence persists even if $\sigma_{i}$ is independent of $\left(\Delta \bar{m}^{2}, \tan ^{2} \bar{\theta}\right)$ (which is true, e.g., for the measured gallium capture rate). In fact, the positive and negative values for $\delta\left(f_{\mathrm{Be}}\right)_{i}$ will generally not be equal.

\section{The accuracy with which the total ${ }^{7}$ Be flux can be determined}

Table IV presents the calculated uncertainties and the best-fit values of $f_{\mathrm{Be}}$ for a representative set of possible neutrino oscillation parameters $\left(\Delta \bar{m}^{2}, \tan ^{2} \bar{\theta}\right)$, that may be obtained from the KamLAND measurements. The largest uncertainty $\sim 22 \%$ is due to the experimental error on the measured gallium rate [14-16]. The two next largest uncertainties, both $\sim 12 \%$, arise from the theoretical calculation of the gallium absorption cross sections and the simulated errors in the KamLAND measurements. The rather large uncertainty due to the gallium cross sections requires explanation since the uncertainties on the individual cross sections are much smaller [44] [e.g., 2.3\% for $p p$ neutrinos and $5 \%$ (average) for ${ }^{7} \mathrm{Be}$ neutrinos]. The amplification in the error due to the cross sections arises because all of the low energy cross section errors are fully correlated. The gallium cross 
TABLE IV. Best-fit values of $f_{\mathrm{Be}}$ and associated uncertainties (gallium based simulation). The table shows the best-fit values and associated uncertainties that are obtained by solving Eq. (23) for a representative set of oscillation parameters within the expected KamLAND and SNO allowed region (see Fig. 1).

\begin{tabular}{|c|c|c|c|c|c|c|c|}
\hline \multirow[b]{2}{*}{$\Delta \bar{m}^{2}$} & \multirow[b]{2}{*}{$\tan ^{2} \bar{\theta}$} & \multirow[b]{2}{*}{$f_{\mathrm{Be}}$} & \multicolumn{5}{|c|}{ Uncertainties $\delta\left(f_{\mathrm{Be}}\right)_{i}(\%)$} \\
\hline & & & $\mathrm{Ga}, \exp$ & Ga, c.s. & KamLAND & CNO (flux) & Total \\
\hline $5.0 \times 10^{-5}$ & $4.2 \times 10^{-1}$ & 1.15 & 22 & $\begin{array}{l}+9 \\
-15\end{array}$ & $\begin{array}{l}+9 \\
-10\end{array}$ & 4.5 & $\begin{array}{l}+25 \\
+28\end{array}$ \\
\hline $5.0 \times 10^{-5}$ & $5.01 \times 10^{-1}$ & 1.31 & 20 & $\begin{array}{l}+9 \\
+13\end{array}$ & $\begin{array}{l}+7 \\
+7\end{array}$ & 4.0 & $\begin{array}{l}+20 \\
+25\end{array}$ \\
\hline $5.0 \times 10^{-5}$ & $2.51 \times 10^{-1}$ & 0.63 & 24 & $\begin{array}{l}13 \\
+15 \\
-22\end{array}$ & $\begin{array}{l}-6 \\
+17 \\
-17\end{array}$ & 8.1 & $\begin{array}{l}+42 \\
-43\end{array}$ \\
\hline $7.94 \times 10^{-5}$ & $4.2 \times 10^{-1}$ & 1.09 & 23 & $\begin{array}{l}+10 \\
+15\end{array}$ & $\begin{array}{l}+14 \\
-13\end{array}$ & 4.8 & $\begin{array}{l}+28 \\
-31\end{array}$ \\
\hline $7.94 \times 10^{-5}$ & $5.01 \times 10^{-1}$ & 1.25 & 20 & $\begin{array}{l}+9 \\
-14\end{array}$ & $\begin{array}{l}+13 \\
-11\end{array}$ & 4.2 & $\begin{array}{l}+26 \\
-27\end{array}$ \\
\hline $7.94 \times 10^{-5}$ & $2.51 \times 10^{-1}$ & 0.58 & 36 & $\begin{array}{l}+16 \\
-24\end{array}$ & $\begin{array}{l}+23 \\
-22\end{array}$ & 9.1 & $\begin{array}{l}+47 \\
+50\end{array}$ \\
\hline $3.16 \times 10^{-5}$ & $4.2 \times 10^{-1}$ & 1.26 & 21 & $\begin{array}{l}-24 \\
+14\end{array}$ & $\begin{array}{l}-22 \\
+8 \\
-7\end{array}$ & 4.0 & $\begin{array}{l}+25 \\
+26\end{array}$ \\
\hline $3.16 \times 10^{-5}$ & $5.01 \times 10^{-1}$ & 1.40 & 19 & $\begin{array}{l}+8 \\
-13\end{array}$ & $\begin{array}{l}+6 \\
+6\end{array}$ & 3.6 & $\begin{array}{l}+20 \\
+24\end{array}$ \\
\hline $3.16 \times 10^{-5}$ & $2.51 \times 10^{-1}$ & 0.73 & 31 & $\begin{array}{l}-13 \\
+13 \\
-16\end{array}$ & $\begin{array}{l}-6 \\
+21 \\
-15\end{array}$ & 6.6 & $\begin{array}{l}-24 \\
+41 \\
-41\end{array}$ \\
\hline
\end{tabular}

section errors are added linearly in calculating $\delta f_{\text {Be }}$ [see Eq. (23)] rather than being combined quadratically.

We have also computed a representative (average) error in the determination of the total ${ }^{7} \mathrm{Be}$ flux by a Monte Carlo sampling of the allowed KamLAND oscillation region shown in Fig. 1. We find

$$
f_{\mathrm{Be}}=f_{\text {Be,best fit }}\left[1.00_{-0.29}^{+0.27}\right] \text {. }
$$

Figure 6 shows the contours of the maximum percentage deviation (in absolute value) from the local best-fit value of $f_{\mathrm{Be}}$. The figure shows that within the currently allowed $1 \sigma$ solar neutrino oscillation region the expected uncertainty in the determination of the ${ }^{7} \mathrm{Be}$ flux is of the order of $25-35 \%$ [in agreement with Eq. (26)].

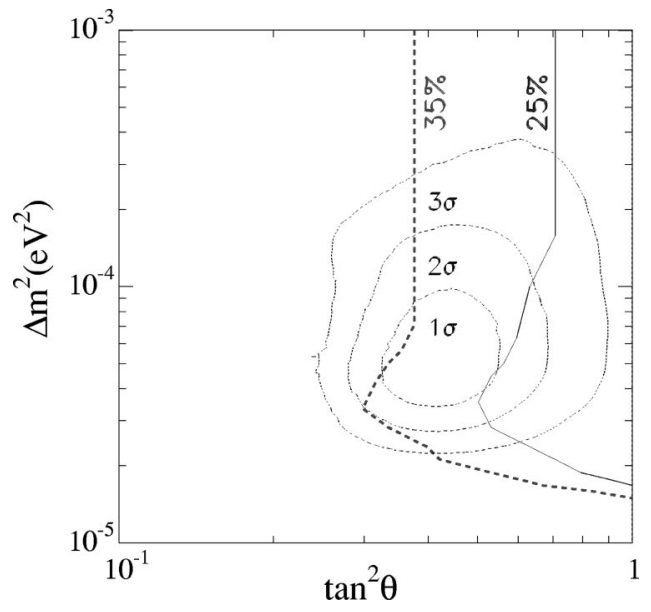

FIG. 6. Percentage error in determining the total ${ }^{7} \mathrm{Be}$ flux. The figure shows two contours (in \%) for the uncertainty in determining the total ${ }^{7} \mathrm{Be}$ flux. The uncertainties were calculated as described in Sec. IV A and Sec. IV B, using a combined analysis of SNO CC and gallium data together with simulated KamLAND data. The curves labeled $1 \sigma, 2 \sigma$, and $3 \sigma$ represent allowed regions from a global analysis of the available solar and reactor data (see Fig. 1).

\section{Can one use the chlorine experiment to determine the ${ }^{7} \mathrm{Be}$ solar neutrino flux?}

We can derive an expression for $f_{\mathrm{Be}}$ in terms of the measured event rate in the chlorine $[12,43]$ solar experiment. Replacing "gallium" by "chlorine" everywhere in Secs. IV A-IV C, we find

$$
\begin{aligned}
f_{\mathrm{Be}}= & \frac{1}{R_{\mathrm{Cl}}^{{ }^{7} \mathrm{Be}, \mathrm{SSM}}\left\langle P_{e e}\left(\Delta \bar{m}^{2}, \tan ^{2} \bar{\theta}\right)_{\mathrm{KamLAND}}\right\rangle_{\mathrm{Cl}}} \\
& \times\left[R_{\mathrm{Cl}}^{\mathrm{exp}}-\sum_{i} R_{\mathrm{Cl}}^{\phi_{i}, \mathrm{SSM}}\left\langle P_{e e}\left(\Delta \bar{m}^{2}, \tan ^{2} \bar{\theta}\right)_{\text {KamLAND }}\right\rangle_{\mathrm{Cl}}^{\phi_{i}}\right. \\
& \left.-R_{\mathrm{Cl}}^{{ }_{\mathrm{B}, \mathrm{SSM}}} \frac{R_{\mathrm{SNO}}^{\mathrm{CC}, \mathrm{exp}}}{R_{\mathrm{SNO}}^{\mathrm{CC}, \mathrm{SSM}}} \frac{\left\langle P_{e e}\left(\Delta \bar{m}^{2}, \tan ^{2} \bar{\theta}\right)_{\text {KamLAND }}\right\rangle_{\mathrm{Cl}}}{\left\langle P_{e e}\left(\Delta \bar{m}^{2}, \tan ^{2} \bar{\theta}\right)_{\text {KamLAND }}\right\rangle_{\mathrm{SNO}}}\right],
\end{aligned}
$$

where $R_{\mathrm{Cl}}^{\mathrm{exp}}=2.56 \pm 0.23 \mathrm{SNU}$ [12]. Equation (27) is the ana$\log$ for the chlorine experiment of the previously derived Eq. (23) that was used in the discussion of extracting $f_{\mathrm{Be}}$ for the gallium experiments.

Table $\mathrm{V}$ lists the best-fit values of $f_{\mathrm{Be}}$ that were obtained by solving Eq. (27) for different pairs of oscillation parameters $\left(\Delta \bar{m}^{2}, \tan ^{2} \bar{\theta}\right)$. The best-fit values of $f_{\mathrm{Be}}$ obtained from the the chlorine experiment are smaller than the best-fit values inferred using the gallium data (see Tables IV and V). Using the chlorine data, one can even get negative (i.e., unphysical) solutions for $f_{\mathrm{Be}}$.

The basic reason for the difficulty in determining $f_{\mathrm{Be}}$ is that the large expected rate from the ${ }^{8} \mathrm{~B}$ neutrino flux, even after reduction due to neutrino oscillations, can account for all the observed rate in the chlorine experiment [46]. The contribution from the ${ }^{7} \mathrm{Be}$ neutrinos is obtained by subtracting the large and somewhat uncertain expected contribution from ${ }^{8} \mathrm{~B}$ neutrinos (and the contributions from $\mathrm{CNO}$ and pep neutrinos that are expected to be much less important) 
TABLE V. Best-fit values of $f_{\mathrm{Be}}$ and associated uncertainties (chlorine based simulation). This table is similar to Table IV except that for Table $\mathrm{V}$ data from the chlorine experiment were used instead of data from the gallium experiment. Because the inferred values of $f_{\mathrm{Be}}$ are small and very uncertain using data from the chlorine experiment, Table $\mathrm{V}$ presents uncertainties as the numerical shift in the best-fit values (not as percentage uncertainties, which are given in Table IV).

\begin{tabular}{ccccccccc}
\hline \hline & & \multicolumn{7}{c}{ Uncertainties $\Delta\left(f_{\mathrm{Be}}\right)_{i}$} \\
\multicolumn{1}{c}{$\Delta \bar{m}^{2}$} & $\tan ^{2} \bar{\theta}$ & $f_{\mathrm{Be}}$ & $\mathrm{Cl}$, exp & $\mathrm{Cl}$, c.s. & SNO, exp & SNO, c.s. & CNO,flux & Total \\
\hline $5.0 \times 10^{-5}$ & $4.2 \times 10^{-1}$ & 0.17 & 0.37 & 0.25 & 0.26 & 0.07 & 0.08 & 0.50 \\
$5.0 \times 10^{-5}$ & $5.01 \times 10^{-1}$ & 0.20 & 0.38 & 0.29 & 0.21 & 0.07 & 0.08 & 0.53 \\
$5.0 \times 10^{-5}$ & $2.51 \times 10^{-1}$ & 0.07 & 0.32 & 0.10 & 0.18 & 0.06 & 0.08 & 0.39 \\
$7.94 \times 10^{-5}$ & $4.2 \times 10^{-1}$ & 0.12 & 0.36 & 0.17 & 0.20 & 0.07 & 0.08 & 0.46 \\
$7.94 \times 10^{-5}$ & $5.01 \times 10^{-1}$ & 0.16 & 0.38 & 0.22 & 0.21 & 0.07 & 0.08 & 0.49 \\
$7.94 \times 10^{-5}$ & $2.51 \times 10^{-1}$ & -0.10 & 0.31 & 0.10 & 0.17 & 0.06 & 0.08 & 0.37 \\
$3.16 \times 10^{-5}$ & $4.2 \times 10^{-1}$ & 0.25 & 0.39 & 0.34 & 0.21 & 0.07 & 0.07 & 0.57 \\
$3.16 \times 10^{-5}$ & $5.01 \times 10^{-1}$ & 0.26 & 0.40 & 0.37 & 0.22 & 0.07 & 0.07 & 0.60 \\
$3.16 \times 10^{-5}$ & $2.51 \times 10^{-1}$ & 0.16 & 0.36 & 0.25 & 0.18 & 0.06 & 0.07 & 0.46 \\
\hline \hline
\end{tabular}

from the total measure chlorine rate. The result is a rather small and uncertain remainder, which can be attributed to ${ }^{7}$ Be neutrinos.

Table $\mathrm{V}$ also presents the most important sources of uncertainty for inferring the value of $f_{\mathrm{Be}}$ using the chlorine, rather than the gallium, data. Since the inferred best-fit values for $f_{\mathrm{Be}}$ that are obtained using the chlorine data are very small (or even negative), we show in Table $\mathrm{V}$ the associated shifts in the prediction of $f_{\mathrm{Be}}$. For the much more reliable inferences from gallium data, we show instead in Table IV the percentage shifts, not the actual numerical shifts.

The largest uncertainties in determining $f_{\mathrm{Be}}$ using the chlorine data are caused by the experimental errors in the chlorine absorption rate $(\sim 0.35)$ and the SNO CC absorption rate $(\sim 0.25)$. The uncertainty in the chlorine neutrino absorption cross sections are also significant $(0.03$ to 0.35 , depending upon the neutrino oscillation parameters). To be explicit, the uncertainties in $f_{\mathrm{Be}}$ due to the theoretical uncertainties in calculating the chlorine neutrino absorption cross sections that were used in constructing Table V were evaluated from the following equation:

$$
\begin{aligned}
\Delta\left(f_{\mathrm{Be}}\right)_{\mathrm{Cl}, \mathrm{c} . \mathrm{s} .}^{2}= & {\left[\sum_{j=p e p,{ }_{\mathrm{Be}, \mathrm{CNO}}}\left[f_{\mathrm{Be}}\left(\text { c.s.s. } \pm 1 \sigma_{\text {c.s. } j}\right)\right]-f_{\mathrm{Be}}(i)\right]^{2} } \\
& +\left[\sum_{j={ }^{8} \mathrm{~B}, h e p}\left[f_{\mathrm{Be}}\left(\text { c.s.s } j \pm 1 \sigma_{\text {c.s. } . j}\right)-f_{\mathrm{Be}}(i)\right]\right]^{2} .
\end{aligned}
$$

(See the Appendix for more details regarding the treatment of the cross section errors.) Even the CNO fluxes $(\sim 0.08)$ and the SNO CC absorption cross section $(\sim 0.10)$ contribute non-negligible errors. The uncertainty from sterile neutrinos is small $(\sim 0.04)$ and does not significantly affect the total uncertainty. Remarkably, the individual uncertainties in $f_{\mathrm{Be}}$ which arise from several different sources are larger than the current best-fit values of $f_{\mathrm{Be}}$ (see Table V).
For the chlorine based determination, we have computed a representative (average) shift in $f_{\mathrm{Be}}$ by a Monte Carlo sampling of the allowed KamLAND oscillation region shown in Fig. 1. We find

$$
\Delta f_{\mathrm{Be}}=0.49,1 \sigma \text {. }
$$

This uncertainty is so large as to render not very useful the determination of $f_{\mathrm{Be}}$ with the aid of chlorine data.

\section{HOW WELL CAN KamLAND PLUS $\nu$ - $e$ SCATTERING EXPERIMENTS DETERMINE THE TOTAL ${ }^{8} B$ AND ${ }^{7}$ Be FLUXES?}

In this section, we show how data from KamLAND can be combined with $\nu$-e scattering data obtained with the Super-Kamiokande and BOREXINO experiments in order to determine, respectively, the total ${ }^{8} \mathrm{~B}$ (Sec. V A) and ${ }^{7} \mathrm{Be}$ (Sec. V B) solar neutrino fluxes. As discussed before, the advantage of using purely $\mathrm{CC}$ measurements to determine the fluxes is that the answers depend only mildly on the active-sterile admixture. On the other hand, $\nu$-e scattering measurements have the advantage of smaller uncertainties in the interaction cross sections. Moreover, for the determination of the ${ }^{7} \mathrm{Be}$ flux, BOREXINO depends less strongly on other neutrino fluxes predicted by the standard solar model than do the radiochemical experiments chlorine, GALLEX, SAGE, and GNO.

\section{A. How well can KamLAND and Super-Kamiokande determine the total ${ }^{8} \mathrm{~B}$ flux?}

In this section, we show how data from the SuperKamiokande and KamLAND experiments can be combined to measure the total ${ }^{8} \mathrm{~B}$ neutrino flux. We shall see that the determination using Super-Kamiokande and KamLAND yields, on average, a value for the total flux than is comparable in precision to what is expected to be obtained using SNO and KamLAND. The systematic uncertainties are dif- 
ferent in the two experiments, SNO and Super-Kamiokande. Therefore, it will be important to compare the total ${ }^{8} \mathrm{~B}$ neutrino flux that is inferred using Super-Kamiokande and KamLAND with the value that is obtained using SNO and KamLAND. If the uncertainties are correctly estimated, then the two methods should agree within their quoted errors.

The advantage of using purely charged current measurements with SNO and KamLAND to determine the total ${ }^{8} \mathrm{~B}$ flux is that the answer depends only very slightly upon the unknown active-sterile mixture (as discussed in Sec. III). The principal advantages of the Super-Kamiokande experiment in the present context is that the neutrino interaction cross section is accurately known [47] and the statistical and systematic errors have already been extensively investigated [5]. However, as we shall see below, when using SuperKamiokande there is a significant uncertainty $(+7$ and $-0 \%)$ in the total ${ }^{8} \mathrm{~B}$ flux due to the active-sterile mixture, at least with our present knowledge of $\eta$.

The average survival probability for ${ }^{8} \mathrm{~B}$ solar neutrinos can be written in the form

$$
\begin{aligned}
\langle P( & \left.\left.\Delta \bar{m}^{2}, \tan ^{2} \bar{\theta}, \eta\right)_{\text {KamLAND }}\right\rangle_{\text {Super-Kamiokande }} \\
= & \left\langle P_{e e}\left(\Delta \bar{m}^{2}, \tan ^{2} \bar{\theta}, \eta\right)_{\text {KamLAND }}\right\rangle_{\text {Super-Kamiokande }} \\
& +\left\langle r P_{e x}\left(\Delta \bar{m}^{2}, \tan ^{2} \bar{\theta}, \eta\right)_{\text {KamLAND }}\right\rangle_{\text {Super-Kamiokande }}
\end{aligned}
$$

where $P$ is the probability of oscillating into active neutrinos and $r \equiv \sigma_{\mu} / \sigma_{e} \simeq 0.15$ is the ratio of the the $\nu_{e^{-e}}$ and $\nu_{\mu}-e$ elastic scattering cross sections. The expected sensitivity of $f_{B}$ to the principal sources of errors can be calculated from the following equation:

$$
\begin{aligned}
\left(\frac{\sigma\left(f_{B}\right)}{f_{B}}\right)^{2}= & \left(\frac{\sigma\left(R_{\text {Super-Kamiokande }}^{\text {exp }}\right)}{R_{\text {Super-Kamiokande }}^{\text {exp }}}\right)^{2} \\
& +\left(\frac{\sigma\left(\langle P\rangle_{\text {Super-Kamiokande,KamLAND }}\right)}{\left(\langle P\rangle_{\text {Super-Kamiokande,KamLAND }}\right)}\right)^{2} .
\end{aligned}
$$

We suppose that KamLAND will observe (with associated uncertainties that we simulate with a Monte Carlo) the rate predicted for the global best-fit point shown in Fig. 1. For purely active oscillations, we find that

$$
f_{\text {active } \mathrm{B}}=1.07\left[1_{-0.028-0.046}^{+0.037+0.040}\right]=1[1 \pm 0.054], \quad \cos ^{2} \eta=1.0,
$$

where the first error corresponds to the Super-Kamiokande experimental uncertainty and the second error is caused by the finite size of the allowed KamLAND region in oscillation parameter space. The combined KamLAND and SuperKamiokande measurement will, for purely active neutrinos, yield a determination for the total ${ }^{8} \mathrm{~B}$ flux that is more accurate than can be obtained with the SNO CC measurement and KamLAND. Within the $1 \sigma$ LMA region, the average uncertainty in the combined KamLAND and SuperKamiokande measurement for the total active ${ }^{8} \mathrm{~B}$ neutrino flux is expected to be

$$
\sigma\left(f_{\text {active B }}\right)=0.06 \text {. }
$$

For purely active neutrinos, Super-Kamiokande and KamLAND may provide us with a more accurate determination of the total ${ }^{8} \mathrm{~B}$ neutrino flux than SNO CC and KamLAND [see Eqs. (19) and (33)].

Allowing for the currently allowed $1 \sigma(25 \%)$ sterile admixture, we find for the best fit point

$$
f_{\text {total B }}=1.07\left[11_{-0.028-0.046-0.00}^{+0.037+0.040+0.07}\right], \quad \cos ^{2} \eta \geqslant 0.75 .
$$

The last error in Eq. (34) corresponds to the present $1 \sigma$ uncertainty from the active-sterile admixture $\cos ^{2} \eta \geqslant 0.75$. The average uncertainty in the combined KamLAND and Super-Kamiokande measurement for $f_{\text {total B }}$ is expected to be ${ }_{-6}^{+9} \%$, within the $1 \sigma$ LMA region and if the sterile admixture is as large as currently allowed.

\section{B. How well can KamLAND and BOREXINO determine the total ${ }^{7} \mathrm{Be}$ flux?}

In this section, we show how data from the KamLAND and BOREXINO experiments can be combined to measure the total ${ }^{7} \mathrm{Be}$ neutrino flux. The principal advantage of using the BOREXINO experiment for this purpose is that the signal in the BOREXINO experiment [40] is predicted to be dominated by ${ }^{7} \mathrm{Be}$ neutrinos, whereas ${ }^{7} \mathrm{Be}$ solar neutrinos are expected to contribute only a relatively small (and unlabeled) fraction to the observed event rate in the gallium and chlorine radiochemical measurements (see Table III). ${ }^{7}$

However, unlike the cases involving the gallium and chlorine experiments that were discussed in Sec. IV, which include only $\nu_{e}(\mathrm{CC})$ absorption, the BOREXINO experiment detects both $\nu_{e^{-}}$scattering and $\nu_{\mu}-e$ and $\nu_{\tau^{-}}$s scattering. One must consider in the present case the extent to which the active-sterile neutrino admixture influences the detected event rate for each set of oscillation parameters $\left(\Delta \bar{m}^{2}, \tan \bar{\theta}^{2}\right)_{\text {KLAND }}$ determined by KamLAND. As we shall see quantitatively in the following discussion, this uncertainty regarding the sterile admixture does not decrease significantly the overall accuracy of the inferred total ${ }^{7} \mathrm{Be}$ neutrino flux.

The average survival probability for active neutrinos can be written conveniently in the form

$$
\begin{aligned}
\left\langle P\left(\Delta \bar{m}^{2}, \tan ^{2} \bar{\theta}, \eta\right)_{\text {KamLAND }}\right\rangle_{\text {BOREXINO }}^{\phi_{i}} & \\
= & \left\langle P_{e e}\left(\Delta \bar{m}^{2}, \tan ^{2} \bar{\theta}, \eta\right)_{\text {KamLAND }}\right\rangle_{\text {BOREXINO }}^{\phi_{i}} \\
& +\left\langle r P_{e x}\left(\Delta \bar{m}^{2}, \tan ^{2} \bar{\theta}, \eta\right)_{\text {KamLAND }}\right\rangle_{\text {BOREXINO }}^{\phi_{i}}
\end{aligned}
$$

where $P^{\phi_{i}}$ is the oscillation probability of neutrino fluxes of source $\phi_{i}$ into active neutrinos and $r \equiv \sigma_{\mu} / \sigma_{e} \simeq 0.15$ is the ratio of the the $\nu_{e}-e$ and $\nu_{\mu}-e$ elastic scattering cross sections.

\footnotetext{
${ }^{7}$ The BOREXINO detector can measure the energy of the recoil electrons produced by $\nu$-e scattering. The radiochemical detectors do not have energy resolution, only an energy threshold.
} 
The expression for $f_{\mathrm{Be}}$ has the same form for the case in which the KamLAND and BOREXINO experiments are considered together as for the previously discussed cases involving the gallium experiments [see Eq. (23)] and the chlorine experiment [see Eq. (27)]. Using the average survival probability defined in Eq. (35), we can write

$$
\begin{aligned}
f_{\mathrm{Be}}= & \frac{1}{R_{\text {BOREXINO }}^{{ }^{7} \mathrm{Be}, \mathrm{SSM}}\left\langle\left(P \Delta \bar{m}^{2}, \tan ^{2} \bar{\theta}, \eta\right)_{\text {KamLAND }}\right\rangle_{\text {BOREXINO }}} \\
& \times\left[R_{\mathrm{BOREXINO}}^{\exp }-\sum_{i} R_{\text {BOREXINO }}^{\phi_{\mathrm{i}}, \text { SSM }}\right. \\
& \left.\times\left\langle P\left(\Delta \bar{m}^{2}, \tan ^{2} \bar{\theta}, \eta\right)_{\text {KamLAND }}\right\rangle_{\text {BOREXINO }}^{\phi_{i}}\right] .
\end{aligned}
$$

In the last term of Eq. (36), we include the contributions from $p p$, and $\mathrm{CNO}$ neutrinos. The contribution from ${ }^{8} \mathrm{~B}$ neutrinos is negligible because the observed ${ }^{8} \mathrm{~B}$ neutrino flux is about a factor of $10^{3}$ smaller than the predicted SSM ${ }^{7} \mathrm{Be}$ neutrino flux and because ${ }^{8} \mathrm{~B}$ neutrinos primarily produce high energy recoils electrons that the BOREXINO detector can distinguish from the low energy recoil electrons produced by ${ }^{7} \mathrm{Be}$ neutrinos.

The expected sensitivity of $f_{\mathrm{Be}}$ to different sources of errors is given by

$$
\begin{aligned}
\left(\frac{\sigma\left(f_{\mathrm{Be}}\right)}{f_{\mathrm{Be}}}\right)^{2}= & \delta\left(f_{\mathrm{Be}}\right)_{\mathrm{BOREXINO}, \exp }^{2}+\delta\left(f_{\mathrm{Be}}\right)_{\mathrm{KamLAND}}^{2} \\
& +\delta\left(f_{\mathrm{Be}}\right)_{\mathrm{CNO}}^{2}+\text { smaller terms. }
\end{aligned}
$$

In order to estimate the accuracy of this method for determining $f_{\mathrm{Be}}$, we suppose that KamLAND will observe (with associated uncertainties that we simulate with a Monte Carlo) the rate predicted for the global best-fit point ${ }^{8}$ shown in Fig. 1 and that BOREXINO also will observe a signal corresponding to this best-fit point (with associated uncertainties). In the absence of any published data, we estimatebased upon experience in previous solar neutrino experiments $[4,5,12,14-16]$ - that BOREXINO will achieve a systematic uncertainty of between 5 and $10 \%$. We estimate in this way that the combined experiments will yield a determination of $f_{\mathrm{Be}}$ that is, for purely active oscillations,

$$
\begin{aligned}
f_{\text {active Be }} & =1.00[1 \pm 0.05(0.1) \pm 0.020 \pm 0.020] \\
& =1[1 \pm 0.057( \pm 0.103)], \quad \cos ^{2} \eta=1.0,
\end{aligned}
$$

where the first error corresponds to the BOREXINO experimental uncertainty, the second to the uncertainty in the reconstructed KamLAND region, and the last error is due to the theoretical uncertainty in the predicted CNO fluxes. Within the $1 \sigma$ LMA region, the average uncertainty in the

\footnotetext{
${ }^{8}$ The predicted rate in units of the expected SSM rate is $R_{\text {BOREXINO }}^{\exp }=0.64$, see Ref. [36].
}

combined KamLAND and BOREXINO measurement for the total active ${ }^{7} \mathrm{Be}$ neutrino flux is expected to be

$$
\sigma\left(f_{\text {active Be }}\right)=0.06(0.105) \text {. }
$$

If we allow for a $25 \%$ sterile admixture, we find

$$
f_{\text {total Be }}=1.00\left[1 \pm 0.05(0.1) \pm 0.02 \pm 0.020_{-0.00}^{+0.05}\right] \text {. }
$$

The last error in Eq. (40) corresponds to the present $1 \sigma$ uncertainty from the active-sterile admixture $\cos ^{2} \eta \geqslant 0.75$. The numbers in parentheses in Eq. (38) and Eq. (40) correspond to assuming the larger systematic uncertainty, $\pm 10 \%$, for the BOREXINO measured rate.

For $f_{\text {total Be }}$, the average uncertainty in the combined KamLAND and BOREXINO measurement is expected to be ${ }_{-6}^{+8}\left({ }_{-10}^{+11}\right) \%$, within the $1 \sigma$ LMA region and if the sterile admixture is as large as currently allowed.

\section{DETERMINATION OF THE $p p$ NEUTRINO FLUX}

In this section, we analyze three strategies for determining the total $p p$ solar neutrino flux without requiring a dedicated experiment that measures only the $p p$ neutrinos. We first describe in Sec. VI A how one can make a crude determination of the $p p$ neutrino flux using the measured Gallium, chlorine, and SNO (CC) event rates $[3,12,15,16]$ and the standard solar model predictions for all but the ${ }^{8} \mathrm{~B},{ }^{7} \mathrm{Be}$, and $p p$ solar neutrino fluxes. This part of the discussion is similar to the analysis described in Ref. [3], although we evaluate explicitly the uncertainty caused by the finite size of the allowed region in oscillation parameter space. We then determine in Sec. VI B how well one can infer the $p p$ flux using just the gallium and the SNO measurements and the BP00 predictions for the other neutrino fluxes, especially the ${ }^{7} \mathrm{Be}$ neutrino flux. The unknown sterile-active mixture contributes only a negligible error using the strategies described in Secs. VI A and VI B. Next, we show in Sec. VI C how the precision of the determination of the $p p$ flux can be improved by using, in the future, the results from KamLAND (to constrain the neutrino oscillation parameters) and from BOREXINO (to constrain the ${ }^{7} \mathrm{Be}$ neutrino flux). The principle of this strategy has also been discussed by the SAGE Collaboration [3].

The theoretical error on the $p p$ neutrino flux is $\pm 1 \%$ (see Ref. [11]), which is an order of magnitude smaller than the estimated errors that we find in this section on the empirically derived values of $f_{p p}$. To achieve a precision of $10 \%$ or better in the determination of the solar neutrino $p p$ flux will require a dedicated and accurate experiment devoted to measuring the $p p$ flux.

Throughout this section, we treat the $p p$ flux and the $p e p$ flux as a single variable because they are so closely linked physically [48]. The two reactions are linked because the pep reaction is obtained from the $p p$ reaction by exchanging a positron in the final state with an electron in the initial state. Thus the rates for the two reactions are proportional to each other to high accuracy [49]. For convenience, we denote the sum of the $p p$ and $p e p$ fluxes as simply $p p$. 


\section{A. Using chlorine, gallium, and SNO data and BP00 predictions}

In this subsection, we show how data from the chlorine, gallium, and SNO experiments can be combined with the $\mathrm{BP} 00$ predictions for the $\mathrm{CNO}$ and hep fluxes to determine the total $p p$ neutrino flux. The reduced $p p$ solar neutrino flux, $f_{p p}$ defined (by analogy with $f_{B}$ ) with respect to the predicted standard solar model sum of the $p p$ and $p e p$ fluxes, can be written as

$$
\begin{aligned}
f_{p p}= & \frac{1}{R_{\mathrm{Ga}}^{p p, \mathrm{SSM}}\left\langle P_{e e}\left(\Delta m^{2}, \tan ^{2} \theta\right)\right\rangle_{\mathrm{Ga}}^{p p}} \\
& \times\left[R_{\mathrm{Ga}}^{\exp }-\sum_{i} f_{i} R_{\mathrm{Ga}}^{\phi_{\mathrm{i}}, \mathrm{SSM}}\left\langle P_{e e}\left(\Delta m^{2}, \tan ^{2} \theta\right)\right\rangle_{\mathrm{Ga}}^{\phi_{i}}\right. \\
& -f_{\mathrm{Be}} R_{\mathrm{Ga}}^{{ }^{7} \mathrm{Be}, \mathrm{SSM}}\left\langle P_{e e}\left(\Delta m^{2}, \tan ^{2} \theta\right)\right\rangle_{\mathrm{Ga}}{ }^{7} \mathrm{Be} \\
& -R_{\mathrm{Ga}}{ }^{{ }_{\mathrm{B}}, \mathrm{SSM} R_{\mathrm{SNO}}^{\mathrm{CC}, \exp }} \frac{\left\langle P_{e e}\left(\Delta m^{2}, \tan ^{2} \theta\right)\right\rangle_{\mathrm{Ga}}{ }^{\mathrm{CC}, \mathrm{SSM}}}{R_{\mathrm{SNO}}} \frac{\left.\left\langle P_{e e}\left(\Delta m^{2}, \tan ^{2} \theta\right)\right\rangle_{\mathrm{SNO}}^{{ }^{\mathrm{B}}}\right] .}{}
\end{aligned}
$$

Here the sum over $i$ in Eq. (41) refers to the three CNO neutrino sources and the hep neutrinos (see Table III).

We insert in Eq. (41) the expression for $f_{\mathrm{Be}}$ in terms of the Chlorine and SNO rates and the standard solar model CNO and hep neutrino fluxes. Explicitly,

$$
\begin{aligned}
f_{\mathrm{Be}}= & \frac{1}{R_{\mathrm{Cl}}{ }^{7} \mathrm{Be}, \mathrm{SSM}}\left\langle P_{e e}\left(\Delta m^{2}, \tan ^{2} \theta\right)\right\rangle_{\mathrm{Cl}}^{{ }^{7} \mathrm{Be}} \\
& \times\left[R_{\mathrm{Cl}}^{\exp }-\sum_{i} f_{i} R_{\mathrm{Cl}}^{\phi_{\mathrm{i}}, \mathrm{SSM}}\left\langle P_{e e}\left(\Delta m^{2}, \tan ^{2} \theta\right)\right\rangle_{\mathrm{Cl}}^{\phi_{i}}\right. \\
& \left.-R_{\mathrm{Cl}}^{{ }^{8} \mathrm{~B}, \mathrm{SSM} R_{\mathrm{SNO}}^{\mathrm{CC}, \mathrm{exp}}} \frac{\left\langle P_{e e}\left(\Delta m^{2}, \tan ^{2} \theta\right)\right\rangle_{\mathrm{Cl}}}{R_{\mathrm{SNO}}^{\mathrm{CC}, \mathrm{SSM}}} \frac{\left\langle P_{e e}\left(\Delta m^{2}, \tan ^{2} \theta\right)\right\rangle_{\mathrm{SNO}}}{\left\langle{ }_{\mathrm{S}}\right.}\right] .
\end{aligned}
$$

For the special case of the best fit point in the LMA solution region, we find

$$
f_{p p}=1.41\left(1 \pm 0.08_{-0.05-0.007}^{+0.03+0.009} \pm 0.06_{-0.19-0.11}^{+0.06+0.04}\right) .
$$

The first error in Eq. (43), $( \pm 0.08)$, results from the weighted average experimental error for the Gallium experiment $[3,15,16]$. The second error $\left({ }_{-0.05}^{+0.03}\right)$ reflects the uncertainties in the calculated neutrino absorption cross sections on gallium [44]. The third error $\left(\begin{array}{l}+0.009 \\ -0.007\end{array}\right)$ is caused by the uncertainties in the calculated standard solar model CNO fluxes [11]. The fourth error $( \pm 0.06)$ derives from the measurement errors in the SNO CC experimental data [1] and neutrino absorption cross section [41]. The fifth error $\left(\begin{array}{l}+0.06 \\ -0.19\end{array}\right)$ results from the experimental error for the chlorine event rate [12] and the sixth error $\left(\begin{array}{c}+0.04 \\ -0.11\end{array}\right)$ reflects the uncertainties in the calculated neutrino absorption cross sections for chlorine $[48,50]$. The physical constraint $f_{\mathrm{Be}} \geqslant 0$ was imposed in obtaining these errors.

Within the $1 \sigma$ LMA allowed region, we find that the central value of $f_{p p}$ can vary in the range $1.32<f_{p p}<1.50$ and the best-fit value can be determined with an average uncertainty of ${ }_{-24}^{+13} \%$. The range of central values of $f_{p p}$ always exceeds the BP00 value because the observed chlorine rate implies a low value of $f_{7} \mathrm{Be}$ Since the largest contributions to the gallium rate are from $p p$ neutrino and ${ }^{7} \mathrm{Be}$ neutrinos, a low ${ }^{7} \mathrm{Be}$ flux implies a relatively high $p p$ flux.

We can summarize the results of the simulations carried out within the $1 \sigma$ allowed LMA region for the $p p$ flux as follows:

$$
f_{p p}=1.41\left(1 \pm 0.06_{-0.24}^{+0.13}\right)=1.41\left(1_{-0.25}^{+0.14}\right) .
$$

The first error in Eq. (44) results from the allowed range of neutrino oscillation parameters and the second error results from the uncertainties in all other recognized sources of errors, combined quadratically. The result given in Eq. (44) should be compared with the quoted estimate by the SAGE Collaboration [3], $f_{p p}=1.29(1 \pm 0.23)$. This agreement is very welcome since the SAGE paper points out that they made "Several approximations... whose nature cannot be easily quantified."

\section{B. Using gallium and SNO data and BP00 predictions}

In this subsection, we determine the range of allowed values for $f_{p p}$ using the BP00 predictions (and uncertainties) for the ${ }^{7} \mathrm{Be}$ neutrinos as well as the $\mathrm{CNO}$ and hep neutrinos. We use in this subsection data only from the gallium and SNO solar neutrino experiments.

Following the same line of reasoning as in section VI A, we find for the best fit point in the LMA allowed oscillation region

$$
f_{p p}=1.05\left(1 \pm 0.11_{-0.08-0.03}^{+0.05+0.02} \pm 0.007_{-0.02}^{+0.04}\right) \text {. }
$$

Just as in Eq. (43), the first error in Eq. (45), ( \pm 0.11$)$, is the experimental error from the weighted average event rate in the gallium experiments, the second error $\left({ }_{-0.08}^{+0.05}\right)$ is due to the neutrino absorption cross section on gallium, the third error $\left({ }_{-0.03}^{+0.02}\right)$ is due to the uncertainties in the BP00 predictions of the CNO neutrino fluxes, the fourth error $( \pm 0.007)$ contains the uncertainty in the SNO CC experimental rate and the calculated absorption cross sections on deuterium. The uncertainty in the BP00 prediction for the ${ }^{7} \mathrm{Be}$ neutrino flux contributes the last error $\left({ }_{-0.02}^{+0.04}\right)$ in Eq. (45).

Within the $1 \sigma$ allowed LMA region, we find the central value of $f_{p p}$ varies in the range $0.93<f_{p p}<1.16$; the best-fit value of $f_{p p}$ can be determined with an average uncertainty of $\pm 14 \%$. We can summarize the determination of $f_{p p}$ as follows:

$$
f_{p p}=1.05\left(1_{-0.11}^{+0.105} \pm 0.14\right)=1.05(1 \pm 0.18),
$$


where the first error is the uncertainty due to the neutrino oscillation parameters and the second error contains the uncertainties from all other sources.

\section{Using BOREXINO, KamLAND, Gallium, and SNO data and $\mathrm{BPOO}$ predictions}

In this subsection, we show how the uncertainty in $f_{p p}$ could be improved by using the KamLAND data to determine the neutrino oscillation parameters and the BOREXINO data, together with the KamLAND oscillation parameters, to constrain $f_{7_{\mathrm{Be}}}$ We start with Eq. (41) but now use $f_{\mathrm{Be}}$ as determined from BOREXINO and KamLAND data [Eq. (36)].

We find that for the best-fit LMA solution (assuming that BOREXINO finds the rate expected for this best fit point)

$$
\begin{aligned}
f_{p p}= & 1.05\left[1 \pm 0.11_{-0.08}^{+0.05+0.02} \pm 0.007 \pm 0.05\right. \\
& \left. \pm 0.02(0.04){ }_{-0.02}^{+0.00}\right] .
\end{aligned}
$$

The first error shown in Eq. (47) ( \pm 0.11$)$ is the experimental error on the measured gallium event rate, the second error $\left({ }_{-0.08}^{+0.05}\right)$ represents the uncertainties from the calculated gallium cross sections. The third error $\left({ }_{-0.02}^{+0.01}\right)$ is from the predicted CNO fluxes, and the fourth error $( \pm 0.007)$ contains the uncertainties from the SNO experimental data and calculated deuterium absorption cross sections. The range of oscillation parameters within the KamLAND reconstructed region gives the fifth error $( \pm 0.05)$ and the sixth error $( \pm 0.02[ \pm 0.04])$ results from the uncertainty in the measured BOREXINO event rate [which we take to be $5 \%$ $(10 \%)]$. The unknown active-sterile admixture (which goes in the direction of lowering $f_{p p}$ ) contributes the last error shown in Eq. (47).

On average, the the precision expected using the Gallium, KamLAND, and SNO experiments is

$$
f_{p p}=1.05[1.0+0.14(0.15)] .
$$

The dominant sources of error in Eq. (48) are the experimental error in the gallium rate and the uncertainties in the calculated gallium absorption cross section.

\section{DISCUSSION AND CONCLUSIONS}

In this section, we review and discuss our principal conclusions. We begin in Sec. VII A by summarizing the experimentallyallowed range that currently exists for the total ${ }^{8} \mathrm{~B}$ solar neutrino flux, taking account of the possibility that sterile neutrinos exist. We then summarize in Sec. VII B how well the total ${ }^{8} \mathrm{~B}$ neutrino flux, and separately the sterile ${ }^{8} \mathrm{~B}$ neutrino flux, can be obtained by combining KamLAND and SNO measurements. Next we describe in Sec. VII C how well the total ${ }^{7} \mathrm{Be}$ flux can be determined using data from the KamLAND, gallium (GALLEX, SAGE, and GNO), and SNO experiments, i.e., using only CC disappearance experiments. In this same subsection, we summarize the measurement accuracy that can be obtained using data from just the KamLAND and the BOREXINO ( $\nu$-e scattering) experi- ments. We describe three strategies (making use of existing and future solar neutrino measurements and guidance from the BP00 solar model) for determining the $p p$ solar neutrino flux without the help of an experiment that measures separately the $p p$ neutrinos. We point out in Sec. VII E (and in the Appendix) that in order to determine the total ${ }^{8} \mathrm{~B}$ or ${ }^{7} \mathrm{Be}$ neutrino flux, and a fortiori to determine the sterile contributions to these fluxes, the correlations among theoretical errors for neutrino absorption cross sections and for solar neutrino fluxes must be treated more accurately than in previous discussions. Section VII F summarizes the main focus of the present paper.

We concentrate on procedures to determine experimentally the total solar ${ }^{8} \mathrm{~B}$ neutrino flux and the total solar ${ }^{7} \mathrm{Be}$ neutrino flux in a universe in which sterile neutrinos might exist. Our methods can work only if the LMA solution of the solar neutrino problem is correct.

The numerical values given here for the precision with which different quantities can be determined are obtained using simulations of how well different experiments may perform. Therefore, the numerical values are intended only as illustrative guides to what may be possible.

\section{A. The currently allowed ${ }^{8} \mathbf{B}$ solar neutrino flux if sterile neutrinos exist}

The combined SNO CC data and the Super-Kamiokande $\nu_{e^{-e}}$ scattering data together yield a widely acclaimed agreement between the ${ }^{8} \mathrm{~B}$ solar neutrino flux that is predicted by the standard solar model and the empirically inferred ${ }^{8} \mathrm{~B}$ neutrino flux. However, this agreement between solar model prediction and solar neutrino measurement is not a unique interpretation of the existing measurements if one allows for the possibility that the incident solar neutrino flux could contain a significant component of sterile neutrinos. We show in Table I and in Sec. II that if one takes account of the possibility that sterile neutrinos may exist then the total solar ${ }^{8} \mathrm{~B}$ neutrino flux could be as large as 2.3 times the flux predicted by the standard solar model. In principle, the existing solar neutrino data could be inconsistent with the standard solar model predictions.

\section{B. Determining the total ${ }^{8} \mathrm{~B}$ solar neutrino flux including sterile neutrinos}

The total ${ }^{8} \mathrm{~B}$ solar neutrino flux, active plus sterile neutrinos, can be determined with a typical $1 \sigma$ accuracy of about $10 \%$ by comparing the charged current measurements from the KamLAND reactor experiment and the SNO experiments (see Sec. III). The active ${ }^{8} \mathrm{~B}$ neutrino flux has been measured by comparing the SNO CC flux (which measures $\nu_{e}$ ) with the Super-Kamiokande neutrino-electron scattering rate (which measures $\nu_{e}$ plus, with less sensitivity, $\nu_{\mu}+\nu_{\tau}$ ). The SNO neutral current measurement will provide an additional and, ultimately, more accurate measurement of the total active ${ }^{8} \mathrm{~B}$ solar neutrino flux.

By subtracting the independently measured active ${ }^{8} \mathrm{~B}$ neutrino flux from the total (active plus sterile) ${ }^{8} \mathrm{~B}$ neutrino flux, one can determine empirically the sterile component of the solar neutrino flux. The active ${ }^{8} \mathrm{~B}$ neutrino flux will even- 
tually be determined accurately by the SNO neutral current measurement $[4,51]$. We estimate therefore that the procedure described here has the potential of measuring, or setting an upper limit on, the sterile component of the ${ }^{8} \mathrm{~B}$ neutrino flux that is as small as $12 \%$ of the total ${ }^{8} \mathrm{~B}$ solar neutrino flux.

The measurement of the total ${ }^{8} \mathrm{~B}$ neutrino flux, and the sterile component of this flux, are independent of solar model considerations. In order to establish the quantitative conclusions, we have performed detailed simulations of the accuracy of the KamLAND reactor experiment in determining neutrino oscillation parameters (see Fig. 1) and have evaluated the theoretical and experimental uncertainties that affect the different flux determinations (see Sec. III C and the Appendix).

The combined measurements of the Super-Kamiokande and KamLAND experiments can be used to determine independently a value for the total ${ }^{8} \mathrm{~B}$ neutrino flux. This determination may be as accurate as $6 \%$ for purely active neutrinos. With the current limits on the active-sterile admixture, the total ${ }^{8} \mathrm{~B}$ neutrino flux could be inferred to an accuracy of $9 \%$ or better, as described in Sec. V A. It will be important to compare the value of the total ${ }^{8} \mathrm{~B}$ neutrino flux inferred by combining the KamLAND and SNO charged current measurements with the value obtained using the KamLAND and Super-Kamiokande experiments. This comparison will be an important test of whether the systematic uncertainties in the experiment and in the analyses are understood.

\section{Determining the total ${ }^{7} \mathrm{Be}$ solar neutrino flux including sterile neutrinos}

The total ${ }^{7} \mathrm{Be}$ solar neutrino flux, active plus sterile neutrinos, can be determined to a $1 \sigma$ accuracy of about $30 \%$ by combining measurements from KamLAND, SNO, and the gallium experiments (see Sec. IV). Unlike the purely empirical determination that is possible for the ${ }^{8} \mathrm{~B}$ flux, the measurement of the total ${ }^{7} \mathrm{Be}$ solar neutrino flux requires some assumption regarding the $\mathrm{CNO}$ solar neutrino fluxes. In our estimates, we have assumed that the standard solar model predictions for the CNO fluxes, and their uncertainties, are at least approximately valid. Table IV shows that as long as the CNO fluxes are not a factor of 3 or more larger than the standard solar model predictions, then they will not significantly limit the accuracy with which the total ${ }^{7} \mathrm{Be}$ neutrino flux can be determined. The measured capture rate in the gallium experiments (GALLEX, SAGE, and GNO) currently constitutes the largest recognized uncertainty in the determination of the total ${ }^{7} \mathrm{Be}$ flux by the method described here (see Table IV). The constraints provided by the chlorine experiment are not very useful in providing an accurate determination of the ${ }^{7} \mathrm{Be}$ neutrino flux [see Table V and Eq. (29)].

One can also determine the allowed range of the total ${ }^{7} \mathrm{Be}$ solar neutrino flux using the data from the KamLAND reactor experiment and the BOREXINO solar neutrino experiment. We show in Sec. V B that with this method one may hope to obtain a $1 \sigma$ accuracy of $11 \%$ or better for the total ${ }^{7} \mathrm{Be}$ solar neutrino flux, which is more accurate than we estimate will be possible with the gallium and chlorine radiochemical experiments.

One can determine an upper limit to the sterile component of the ${ }^{7} \mathrm{Be}$ solar neutrino flux by combining the measured rate in the neutrino-electron scattering experiment BOREXINO with the ${ }^{7} \mathrm{Be}$ total flux inferred from measurements of the KamLAND, SNO, and gallium experiments. If one assumes that the entire signal measured in BOREXINO is due to $\nu_{e}$, then one obtains a minimum value for the active component of the ${ }^{7} \mathrm{Be}$ neutrino flux. Subtracting this minimum value from the total ${ }^{7} \mathrm{Be}$ flux, one will obtain an upper limit to the sterile component of the flux. It seems unlikely that the procedure described here has the sensitivity to measure a value for the sterile component unless the sterile flux is larger than $30 \%$ of the total ${ }^{7} \mathrm{Be}$ neutrino flux. However, limits on the sterile neutrino admixture can be obtained from the analysis of ${ }^{8} \mathrm{~B}$ and KamLAND neutrino measurements described in Sec. VII B.

In order to make a direct and precise measurement of the sterile component of the ${ }^{7} \mathrm{Be}$ solar neutrino flux, we need a charged current measurement of the ${ }^{7} \mathrm{Be}$ flux. A ${ }^{7} \mathrm{Be}$ solar neutrino absorption experiment, e.g., with a lithium target [52] or with LENS [53], would make possible an accurate determination of the sterile ${ }^{7} \mathrm{Be}$ neutrino flux by providing a set of experimental constraints that is analogous to what will exist for the SNO, Super-Kamiokande, and KamLAND experiments.

\section{Determining the $p p$ neutrino flux}

The theoretical uncertainty in the calculated $p p$ solar neutrino flux is estimated to be only $1 \%$ [11]. Therefore, a precise determination of the $p p$ solar neutrino flux will be of great interest as a crucial test of the theory of stellar evolution. The measurement of the $p p$ neutrino flux will also provide a critical test of whether the neutrino oscillation theory, which works well at energies above $5 \mathrm{MeV}$, also describes accurately the lower energy neutrino phenomena (energies less than $0.4 \mathrm{MeV}$ ). We show in Sec. VI that the $p p$ flux can be determined with modest accuracy, of order 18 to $20 \%$, using a combination of existing experimental data and some guidance from the BP00 standard solar model. In the future, it should be possible to determine the $p p$ neutrino flux to an accuracy of $15 \%$ using experimental data from the BOREXINO, KamLAND, and SNO solar neutrino experiments and predictions (in a noncritical way) from the standard solar model [see Eq. (48)]. The unknown flux of sterile neutrinos does not significantly affect the quoted estimates on the accuracy with which the $p p$ flux can be determined. To measure the $p p$ flux with an accuracy sufficient to test stringently the standard solar model prediction will require a dedicated and accurate experiment that measures separately the $p p$ neutrino flux.

\section{E. Correlation of errors, especially for neutrino absorption cross sections}

In order to determine the total ${ }^{8} \mathrm{~B}$ or ${ }^{7} \mathrm{Be}$ solar neutrino flux, and their sterile components, one must evaluate care- 
fully all known sources of error. In the course of this investigation, we realized that the evaluations of the neutrino absorption cross section uncertainties in previous neutrino oscillation studies, including our own, have not properly taken account of the correlations among the theoretical uncertainties in the cross section calculations (for an insightful discussion of this point, see Ref. [54]). We discuss in the Appendix how the cross section errors can be treated more correctly. We also emphasize here that it is necessary to treat as fully correlated the uncertainties in the principal $\mathrm{CNO}$ neutrino fluxes that are obtained from standard solar model predictions. These effects are small compared to other uncertainties in determining solar neutrino oscillation parameters (the principal goal of nearly all previous oscillation studies). The correlations among cross section uncertainties become important only when one wants to make accurate inferences regarding the neutrino fluxes themselves.

\section{F. Focus: total fluxes and sterile neutrino fluxes}

The main focus in this paper is on determining experimentally the total ${ }^{8} \mathrm{~B},{ }^{7} \mathrm{Be}$, and $p p$ solar neutrino fluxes in order to make possible more precise tests of solar model predictions. We have also shown that the contribution of sterile neutrinos to the total flux can be measured, or a useful upper limit can be set, for ${ }^{8} \mathrm{~B}$ solar neutrinos and for ${ }^{7} \mathrm{Be}$ solar neutrinos.

\section{ACKNOWLEDGMENTS}

J.N.B. acknowledges support from NSF Grant No. PHY0070928. M.C.G.-G. was supported by the European Union Grant No. HPMF-CT-2000-00516. This work was also supported by the Spanish DGICYT under Grants No. PB98-0693 and FPA2001-3031.

\section{APPENDIX}

We determine, as is conventional for many analyses of solar neutrino data, the allowed regions in the neutrino oscillation space using a $\chi^{2}$ function that includes all the relevant data. In the construction of the $\chi^{2}$ function, we have followed closely the prescription of Ref. [55] (see also Ref. [54]), but we have included some modifications to this prescription in order to account in more detail for the energy dependence and the correlation of the cross section errors for the chlorine and gallium solar neutrino experiments.

For a given experiment $j$ (for example, gallium, or chlorine), the expected number of events can be written as

$$
R_{j}=\sum_{i=1}^{8} \sum_{k} \phi_{i}\left(E_{k}\right) C_{j}\left(E_{k}\right) P_{e e, i}\left(E_{k}, \Delta m^{2}, \theta\right) \equiv \sum_{i=1}^{8} R_{i j}
$$

where $i=1,8$ labels the solar neutrino fluxes, $\phi_{i}$, and $k$ labels the energy bins of energy $E_{k}$. The quantity $C_{j}\left(E_{k}\right)$ is the cross section for the interaction of a neutrino of energy $E_{k}$ in the experiment $j ; P_{e e, i}\left(E_{k}, \Delta m^{2}, \theta\right)$ is the survival probability of $\nu_{e}$ for a given energy $E_{k}$ at a specified point, $\Delta m^{2}, \theta$, in neutrino oscillation space. ${ }^{9}$

The error matrix for the neutrino absorption cross sections can be derived using Eq. (A1). Since the errors are uncorrelated between different experiments, we need to evaluate the following expression for a specified experiment:

$$
\sigma^{2}(\text { c.s. })=\left\langle\left(R-R^{0}\right)^{2}\right\rangle
$$

where $R^{0}$ is the best estimate for the (gallium or chlorine) experimental capture rate and the brackets indicate an average over the probability distribution of uncertainties in the neutrino cross sections. Writing out the various terms in Eq. (A2), we find

$$
\begin{aligned}
\sigma^{2}(\text { c.s. })= & \sum_{i, j} \sum_{k 1, k 2} \phi_{i}\left(E_{k 1}\right) \phi_{j}\left(E_{k 2}\right) P_{i}\left(E_{k 1}\right) P_{j}\left(E_{k 2}\right) \\
& \times\left\langle\Delta C\left(E_{k 1}\right) \Delta C\left(E_{k 2}\right)\right\rangle .
\end{aligned}
$$

In the standard Ref. [55], the cross section error matrix for each experiment is given as

$$
\begin{aligned}
\sigma^{2}(\text { c.s. })_{j j} & =\delta_{i 1, i 2} \sum_{i 1} \sum_{i 2} R_{i 1 j} R_{i 2 j} \Delta \ln C_{i 1}^{j} \Delta \ln C_{i 2}^{j} \\
& =\sum_{i} R_{i j}^{2}\left(\Delta \ln C_{i}^{j}\right)^{2}
\end{aligned}
$$

where $\Delta \ln C_{i}$ is the average uncertainty in the interaction cross section of neutrinos of flux type $i$ in experiment $j$.

Equation (A4) is obtained from Eq. (A1) by neglecting the correlations of the cross section errors among the different neutrino fluxes and by neglecting the energy dependence of the cross section errors. For the gallium detector, neither of these assumptions is correct and even for chlorine the cross section errors for different neutrino sources are strongly correlated.

For all but the ${ }^{8} \mathrm{~B}$ and hep neutrino fluxes, either ground state to ground state transitions are the only energetically possible transitions (which is the case for the chlorine detector) or the ground state to ground state transitions dominate (which is the case for the gallium detector). Thus all the cross sections for the lower energy neutrinos move up or down together, proportional to the square of the dominant matrix element.

When considering the gallium experiments, we include the energy dependence of the cross section errors and assume full correlations among the cross section errors of the different neutrino sources that contribute at a specific energy. Specifically, we use the results from Ref. [56] for the cross section errors. For continuum sources, we take the fractional error of the interaction cross section in the energy bin $k$ to be

\footnotetext{
${ }^{9}$ The survival probability depends upon the neutrino species $i$ because different neutrino species have different probability distributions for the location of their production within the Sun. For matter oscillations, the survival probability obviously depends upon where in the Sun the neutrino was produced.
} 


$$
\Delta \ln C_{\mathrm{Ga}, \mathrm{k}}=\frac{1}{3}\left[\frac{\left|\sigma_{\text {max }, \min }\left(E_{k}\right)-\sigma_{\text {best }}\left(E_{k}\right)\right|}{\sigma_{\text {best }}\left(E_{k}\right)}\right],
$$

where $\sigma_{\text {max } \min }\left(E_{k}\right)$ are the $3 \sigma$ upper and lower limit cross sections given in Tables III and IV of Ref. [56]. For the line sources ${ }^{7} \mathrm{Be}$ and $p e p$ we use the errors given in Eqs. (41) and (42) of Ref. [56]. To be complete, we have checked that the shapes of these neutrino lines (see Ref. [45]) do not affect significantly the error estimates. The gallium contribution to the cross section error matrix is therefore given by

$$
\begin{aligned}
\sigma_{\mathrm{Ga}, \mathrm{Ga}}^{2}(\text { c.s. })= & \sum_{k 1} \sum_{k 2} \frac{\partial R}{\partial \ln C\left(E_{k 1}\right)} \frac{\partial R}{\partial \ln C\left(E_{k 2}\right)} \\
& \times \Delta \ln C(k 1) \Delta \ln C(k 2) \rho_{k 1 k 2},
\end{aligned}
$$

where $\rho_{k 1 k 2}$ is the correlation matrix for the cross section errors of the different energy bins. For low energy neutrino sources, the dominant process is the transition to the germanium ground state and for higher energy neutrino sources $(E \gtrsim 2 \mathrm{MeV})$ transitions to excited states dominate. Therefore, we assume the cross section errors to be fully correlated between energy bins either below or above $E=2 \mathrm{MeV}$ $\left(\rho_{k 1 k 2}=1\right.$ for $E_{k 1}, E_{k 2}<2 \mathrm{MeV}$ or $\left.E_{k 1}, E_{k 2}>2 \mathrm{MeV}\right)$. We take the errors to be uncorrelated between one energy bin below $2 \mathrm{MeV}$ and one energy bin above $E=2 \mathrm{MeV}\left(\rho_{k 1 k 2}\right.$ $=0$ for $E_{k 1}<2 \mathrm{MeV}$ and $E_{k 2}>2 \mathrm{MeV}$, or $E_{k 1}<2 \mathrm{MeV}$ and $\left.E_{k 2}>2 \mathrm{MeV}\right)$.
For chlorine, we take as fully correlated the cross section errors for the $p p$, pep, ${ }^{7} \mathrm{Be}$, and $\mathrm{CNO}$ neutrinos. The cross section errors for the ${ }^{8} \mathrm{~B}$ and hep neutrinos are uncorrelated with the errors for the lower energy neutrinos but are fully correlated with each other. We can neglect for chlorine the energy dependence of the chlorine to argon cross section errors because the uncertainty for the lower energy neutrinos is determined almost entirely from the ground-state to ground-state matrix element. (Forbidden corrections are unimportant for these low energy neutrinos.) For the ${ }^{8} \mathrm{~B}$ and hep neutrinos, the absorption cross sections are also dominated by a single (but different) transition, in this case the superallowed transition [10], and therefore we can also neglect the energy dependence for the higher energy neutrinos. We adopt the values of the averaged chlorine cross section errors given in Refs. $[48,50]$. To be explicit, the chlorine contribution to the cross section error matrix is given by

$$
\sigma_{\mathrm{Cl}, \mathrm{Cl}}^{2}(\text { c.s. })=\sum_{i 1} \sum_{i 2} R_{i 1 \mathrm{Cl}} R_{i 2 \mathrm{Cl}} \Delta \ln C_{i 1}^{\mathrm{Cl}} \Delta \ln C_{i 2}^{\mathrm{Cl}} \rho_{i 1 i 2},
$$

where $\rho_{i 1 i 2}=1$ for $i 1, i 2=p e p, \quad \mathrm{CNO},{ }^{7} \mathrm{Be}$ or $i 1, i 2$ $={ }^{8} \mathrm{~B}$, hep. Also, $\rho_{i 1 i 2}=0$ for $i 1=p e p, \mathrm{CNO},{ }^{7} \mathrm{Be}$ and $i 2$ $={ }^{8} \mathrm{~B}$, hep.
[1] Q.R. Ahmad et al., SNO Collaboration, nucl-ex/0204008.

[2] Q.R. Ahmad et al., SNO Collaboration, nucl-ex/0204009.

[3] SAGE Collaboration, J.N. Abdurashitov et al., astro-ph/0204245.

[4] SNO Collaboration, Q.R. Ahmad et al., Phys. Rev. Lett. 87, 071301 (2001).

[5] Super-Kamiokande Collaboration, S. Fukuda et al., Phys. Rev. Lett. 86, 5651 (2001).

[6] G.L. Fogli, E. Lisi, D. Montanino, and A. Palazzo, Phys. Rev. D 64, 093007 (2001).

[7] C. Giunti, Phys. Rev. D 65, 033006 (2002); M.V. Garzelli and C. Giunti, ibid. 65, 093005 (2002).

[8] G. Fiorentini, F.L. Villante, and B. Ricci, hep-ph/0109275.

[9] J.N. Bahcall, Phys. Rev. C 65, 015802 (2002).

[10] J.N. Bahcall, Phys. Rev. Lett. 12, 300 (1964); R. Davis, Jr., ibid. 12, 303 (1964); J.N. Bahcall and R. Davis, Science 191, 264 (1976)

[11] J.N. Bahcall, M.H. Pinsonneault, and S. Basu, Astrophys. J. 555, 990 (2001).

[12] B.T. Cleveland, T. Daily, R. Davis, Jr., J.R. Distel, K. Lande, C.K. Lee, and P.S. Wildenhain, Astrophys. J. 496, 505 (1998).

[13] Kamiokande Collaboration, Y. Fukuda et al., Phys. Rev. Lett. 77, 1683 (1996).

[14] SAGE Collaboration, J.N. Abdurashitov et al., Phys. Rev. C 60, 055801 (1999).

[15] GALLEX Collaboration, W. Hampel et al., Phys. Lett. B 447, 127 (1999).
[16] E. Belloti, Nucl. Phys. B (Proc. Suppl.) 91, 44 (2001) (http:// nu2000. sno. laurentian. ca).

[17] J.N. Bahcall, S. Basu, and M. Pinsonneault, Phys. Lett. B 433, 1 (1998).

[18] W. Kwong and S.P. Rosen, Phys. Rev. D 54, 2043 (1996).

[19] F.L. Villante, G. Fiorentini, and E. Lisi, Phys. Rev. D 59, 013006 (1999).

[20] J.N. Bahcall, M.C. Gonzalez-Garcia, and C. Pena-Garay, J. High Energy Phys. 08, 014 (2001).

[21] P.I. Krastev and A.Y. Smirnov, Phys. Rev. D 65, 073022 (2002).

[22] J.N. Bahcall, M.C. Gonzalez-Garcia, and C. Pena-Garay, J. High Energy Phys. 04, 007 (2002).

[23] A.R. Junghans, E.C. Mohrmann, K.A. Snover, T.D. Steiger, E.G. Adelberger, J.M. Casandjian, H.E. Swanson, L. Buchmann, S.H. Park, and A. Zyuzin, Phys. Rev. Lett. 88, 041101 (2002).

[24] S.M. Bilenky, C. Giunti, and W. Grimus, Prog. Part. Nucl. Phys. 43, 1 (1999).

[25] M.C. Gonzalez-Garcia and Y. Nir, hep-ph/0202058.

[26] V. Barger, D. Marfatia, and K. Whisnant, Phys. Rev. Lett. 88, 011302 (2002); Phys. Lett. B 509, 19 (2001).

[27] D. Dooling, C. Giunti, K. Kang, and C.W. Kim, Phys. Rev. D 61, 073011 (2000).

[28] C. Giunti, M.C. Gonzalez-Garcia, and C. Pena-Garay, Phys. Rev. D 62, 013005 (2000).

[29] LSND Collaboration, J. Aguilar et al., Phys. Rev. D 64, 112007 (2001). 
[30] KARMEN Collaboration, K. Eitel, Nucl. Phys. B (Proc. Suppl.) 91, 191 (2001).

[31] KamLAND Collaboration, A. Piepke, Nucl. Phys. B (Proc. Suppl.) 91, 99 (2001); J. Busenitz, et al., KamLAND Collaboration (unpublished). May be downloaded from: http:// kamland. lbl. gov/; K. Ishihara for the KamLAND Collaboration, talk at the NuFACT'01 Workshop in Tsukuba, Japan 2001. Transparencies at http://psux1. kek. jp/ nufact01/index. html.

[32] A. Bandyopadhyay, S. Choubey, S. Goswami, and K. Kar, Phys. Lett. B 519, 83 (2001).

[33] V. Barger, D. Marfatia, and B.P. Wood, Phys. Lett. B 498, 53 (2001).

[34] R. Barbieri and A. Strumia, J. High Energy Phys. 12, 016 (2000).

[35] H. Murayama and A. Pierce, Phys. Rev. D 65, 013012 (2002).

[36] J.N. Bahcall, M.C. Gonzalez-Garcia, and C. Pena-Garay, hep-ph/0204314.

[37] M. Smy, Proceedings of the NOON2001 Workshop, Kashiwa, Japan, 2001, hep-ex/0202020.

[38] A. de Gouvea and C. Pena-Garay, Phys. Rev. D 64, 113011 (2001).

[39] M.C. Gonzalez-Garcia and C. Pena-Garay, Phys. Lett. B 527, 199 (2002).

[40] Borexino Collaboration, G. Alimonti et al., Astropart. Phys. 16, 205 (2002); KamLAND Collaboration, A. Piepke et al., Nucl. Phys. B (Proc. Suppl.) 91, 99 (2001).

[41] S. Nakamura, T. Sato, S. Ando, T.-S. Park, F. Myhrer, V. Gudkov, and K. Kubodera, nucl-th/0201062; see also, A. Kurylov, M.J. Ramsey-Musolf, and P. Vogel, Phys. Rev. C 65, 055501
(2002); J.F. Beacom and S.J. Parke, Phys. Rev. D 64, 091302 (2001).

[42] M. Butler, J.-W. Chen, and X. Kong, Phys. Rev. C 63, 035501 (2001).

[43] R. Davis, Jr., D.S. Harmer, and K.C. Hoffman, Phys. Rev. Lett. 20, 1205 (1968).

[44] J.N. Bahcall, Phys. Rev. C 56, 3391 (1997).

[45] J.N. Bahcall, Phys. Rev. Lett. 71, 2369 (1993); Phys. Rev. D 49, 3923 (1994).

[46] J.N. Bahcall and H.A. Bethe, Phys. Rev. Lett. 65, 2233 (1990).

[47] J.N. Bahcall, M. Kamionkowski, and A. Sirlin, Phys. Rev. D 51, 6146 (1995).

[48] J.N. Bahcall, Neutrino Astrophysics (Cambridge University Press, Cambridge, 1989).

[49] J.N. Bahcall and R.M. May, Astrophys. J. 155, 501 (1969).

[50] J.N. Bahcall, E. Lisi, D.E. Alburger, L. De Braeckeleer, S.J. Freedman, and J. Napolitano, Phys. Rev. C 54, 411 (1996).

[51] J.N. Bahcall, P.I. Krastev, and A.Y. Smirnov, Phys. Rev. D 62 , 093004 (2000).

[52] M. Galeazzi, G. Gallinaro, F. Gatti, P. Meunier, S. Vitale, A.V. Kopylov, V.V. Petukhov, E.A. Yanovich, and G.T. Zatsepin, Phys. Lett. B 398, 187 (1997); A. Kopylov and V. Petukhov, hep-ph/0201042.

[53] R.S. Raghavan, Phys. Rev. Lett. 78, 3618 (1997); R.S. Raghavan, hep-ex/0106054.

[54] M.V. Garzelli and C. Giunti, Phys. Lett. B 488, 339 (2000).

[55] G.L. Fogli and E. Lisi, Astropart. Phys. 3, 185 (1995) with updated uncertainties as discussed in G.L. Fogli, E. Lisi, D. Montanino, and A. Palazzo, Phys. Rev. D 62, 013002 (2000).

[56] J.N. Bahcall, Phys. Rev. C 56, 3391 (1997). 